\title{
Spoofing and Price Manipulation in Order Driven Markets
}

\author{
Álvaro Cartea ${ }^{\mathrm{a}}$, Sebastian Jaimungal ${ }^{\mathrm{b}}$, Yixuan Wang $^{1}$ \\ ${ }^{a}$ Mathematical Institute, University of Oxford, Oxford, UK \\ Oxford-Man Institute of Quantitative Finance, Oxford, UK \\ ${ }^{b}$ Department of Statistical Sciences, University of Toronto, Toronto, Canada
}

\begin{abstract}
We model the trading strategy of an investor who spoofs the limit order book (LOB) to increase the revenue obtained from selling a position in a security. The strategy employs, in addition to sell limit orders (LOs) and sell market orders (MOs), a large number of spoof buy LOs to manipulate the volume imbalance of the LOB. Spoofing is illegal, so the strategy trades off the gains that originate from spoofing against the expected financial losses due to a fine imposed by the financial authorities. As the expected value of the fine increases, the investor relies less on spoofing, and if the expected fine is large enough, it is optimal for the investor not too spoof the LOB because the fine outweighs the benefits from spoofing. The arrival rate of buy MOs increases because other traders believe that the spoofed buy-heavy LOB shows the true supply of liquidity and interpret this imbalance as an upward pressure in prices. When the fine is low, our results show that spoofing considerably increases the revenues from liquidating a position. The PnL of the spoof strategy is higher than that of a no-spoof strategy for two reasons. First, the investor employs fewer MOs to draw the inventory to zero and benefits from roundtrip trades, which stem from spoof buy LOs that are 'inadvertently' filled and subsequently unwound with sell LOs. Second, the midprice trends upward when the book is buy-heavy, therefore, as time evolves, the spoofer sells the asset at better prices (on average).
\end{abstract}

Keywords: Market making; Execution; Spoofing; Layering; High-frequency trading; Market quality

\section{Introduction}

In order-driven markets, 'spoofing' and 'layering' are strategies that provide false information about the demand and supply of an asset. These trading strategies are illegal and profit from market participants who trade on misleading market signals. The Dodd-Frank Act describes spoofing as "bidding or offering with the intent to cancel the bid or offer before execution," see Dodd-Frank (2010). Similar to spoofing, layering consists in submitting a relatively large number of orders to one side of the limit order book (LOB) to precipitate an unwarranted change in the price of the asset. Layering and spoofing may

Email addresses: Alvaro.Cartea@maths.ox.ac.uk (Álvaro Cartea), sebastian.jaimungal@utoronto.ca (Sebastian Jaimungal), yixuan.wang@maths.ox.ac.uk (Yixuan Wang) 
cause prices to change because the market interprets the one-sided pressure in the LOB as a shift in the balance of the number of investors who wish to purchase or sell the asset, which causes prices to increase (more buyers than sellers) or prices to decline (more sellers than buyers) ${ }^{1}$

Spoofing and layering are very difficult to detect. These strategies are camouflaged behind the vast number of updates in the LOB and use sophisticated automated algorithms to avoid detection. Nevertheless, regulators and financial authorities have successfully prosecuted many traders for market abuse strategies and price manipulation as a result of spoofing and layering, e.g., see Agency for the Cooperation of Energy Regulators (2019b) $\mathrm{L}^{2}$

We show how an investor employs spoof limit orders (LOs) to manipulate the volume imbalance of the LOB to trade at more advantageous prices. We compute volume imbalance of the LOB as the ratio of volume posted at the best bid price minus the volume posted at the best ask price to the sum of the volume at the best bid and best ask prices. We assume that volume imbalance follows a Markov chain to describe three regimes of the LOB: buy-heavy, neutral, and sell-heavy. For a selection of stocks traded in Nasdaq, we show that the arrival rate of buy market orders (MOs) is highest in the buy-heavy regime, second-highest in the neutral regime, and lowest in the sell-heavy regime. The opposite pattern is found for the arrival rate of sell MOs, see Cartea et al. (2018).

The investor's objective is to maximise the expected profit and loss (PnL) from liquidating a position in shares. The strategy employs LOs and MOs to trade shares, and penalises inventory holdings throughout the trading horizon. Although the objective is to liquidate shares, the investor may employ spoof buy LOs to tilt the volume imbalance in the LOB from sell-heavy and neutral, into the buy-heavy regime - if the LOB is already buy-heavy there is no need to spoof the book. When the book is tilted into the buy-heavy regime, the arrival rate of buy MOs increases and the price of the asset exhibits a positive trend.

In our model, the investor pays a fine if the financial authorities detect spoofing in the LOB and manipulation of prices. The investor's strategy trades off the gains that originate from spoofing against the expected financial losses due to the fine. As the expected value of the fine increases, the investor relies less on spoofing, and if the expected fine is large enough, it is optimal for the investor not too spoof the LOB because the fine outweighs the benefits from spoofing.

Compared with a no-spoof strategy, when the fine for spoofing is low, we show that the investor's spoof strategy liquidates (on average) more shares using sell LOs and relies on fewer MOs to sell shares and to stay on target throughout the trading horizon. Sell MOs receive worse prices than sell LOs because the former are executed at the midprice minus half the quoted spread and incur liquidity taking fees, while the latter are filled at the midprice plus half the quoted spread and liquidity making rebates.

\footnotetext{
${ }^{1}$ See https://www.fca.org.uk/news/statements/statement-regarding-swift-trade-court-appeal-judgment .

${ }^{2}$ See also the cases described in the U.S. Commodity Futures Trade Commission (www.cftc.gov) and the UK's Financial Conduct Authority (www.fca.org.uk).
} 
In addition to the risk of being prosecuted by financial authorities, spoofers bear the risk that their spoof buy LOs could be filled and there may be little time left to employ sell LOs to unwind the 'inadvertently' acquired shares. The probability that a spoof buy LO receives full or partial execution depends on the arrival rate of sell MOs when the LOB is in the buy-heavy regime and on the volume of the spoof buy LO posted at the best bid price to tilt the book. For example, a sell-heavy book requires more spoof buy LOs than a book in the neutral regime to tilt it into the buy-heavy regime.

We use Nasdaq high-frequency data to estimate the parameters of the model and use simulations to analyse the performance of the optimal liquidation strategy for an investor who spoofs the LOB. In one of the examples we provide, we assume that the investor needs to liquidate 30 lots of shares of Intel Corporation, ticker symbol INTC, over a period of 300 seconds. Each lot consists of 300 shares, which is approximately the average volume the LOs posted at the best bid in the LOB of INTC. If the investor does not spoof the LOB, the strategy employs, on average, 24 lots of sell MOs and 6 lots of (filled) sell LOs to liquidate the position.

In the extreme case where there is no penalty for spoofing the book, and market participants believe that the buy-heavy LOB conveys truthful information about demand and supply of the asset, the spoofing strategy liquidates the initial position with an average of: 15 lots of sell MOs, 24 lots of (filled) sell LOs, and 9 lots of (filled) spoof buy LOs. In this example, the difference between the mean PnL received by the investor when the strategy spoofs the LOB and when it does not spoof the book to liquidate an initial target of 9,000 shares is $\$ 12.13 \times 300$. This extra revenue, which stems from employing spoof LOs in the strategy, is approximately $1,213 \times 300$ times the quoted spread in INTC.

In general, the PnL of the spoof strategy is higher than that of a no-spoof strategy for two reasons. First, the investor employs fewer MOs to draw the inventory to zero and benefits from roundtrip trades which stem from spoof buy LOs that are 'inadvertently' filled and subsequently unwound with sell LOs. Second, the midprice trends upward when the book is buy-heavy, therefore, as time evolves, the spoofer sells the asset at better prices (on average).

The spoofing strategy deviates the price of the asset from its fundamental value. Our simulations show that once the investor finalises the liquidation programme, which lasts five minutes, the mean price of the asset is approximately 52 cents higher than the mean price of the asset in the absence of spoofing. The effects of manipulating the price with spoof LOs are expected to subside, so the price of the asset will return to its fundamental value. In the meantime, however, market participants make trading decisions on distorted information about the price of the asset, which may also have a knock-on effect on other assets in the marketplace.

The literature on spoofing is scant. Allen and Gale (1992) propose a simple model for trade-based stock-price manipulation from a uninformed speculator. They show that manipulation is profitable. Lee et al. (2013) define a spoof LO order as an order resting in the book at least 6 ticks away from the market price (in our analysis, the investor posts spoof LOs at the best bid) and the volume of the spoof order is a least twice as large as the average volume of the LOs posted the previous day. They use a proprietary data set with account information from the Korea Exchange. They show empirically 
that the spoof orders create the impression of imbalance in the LOB, which moves the price, and also show that the probability of filling a spoof order is very low. They also show empirically that spoofing achieves substantial extra profits and that spoofing tends to target stocks with: higher volatility of returns, lower market capitalisation, lower price level, and lower managerial transparency. Wang (2015) uses data from the Taiwan Futures Exchange to show that market participants spoof the LOB in the stocks that exhibit high volumes of trading, high volatility, and high prices. The author also shows that spoofing increases the volume of trading, increases the volatility of prices, and increases the quoted spread. In a recent article, the Agency for the Cooperation of Energy Regulators (2019a) investigates spoofing and layering in wholesale energy markets and suggests a list of indicators to identify these illegal activities. There are other studies that look at price manipulation in other contexts, see for example Alfonsi and Acevedo (2014) and Klöck et al. (2017).

Finally, there are studies that employ machine learning algorithms to detect manipulation in markets. Cao et al. (2014) use k-Nearest Neighbour (kNN) and One Class Support Vector Machine (OCSVM) on transformed LOB data to detect price manipulation. They show that most non-stationary features of the data can be removed by transformations based on techniques of time series analysis such as the autoregressive integrated moving average model. Their results indicate that kNN and OCSVM work effectively to detect spoofing and the transformations of the data also improve the power of machine learning algorithms to detect spoofing - the performance of detection is measured in terms of area under Receiver Operating Characteristic (ROC) curve. Cao et al. (2015) use Adaptive Hidden Markov Model with Anomaly States (AHMMAS) to detect manipulation activities in level 2 data from Nasdaq and the London Stock Exchange, and compare the performance of detecting price manipulation with standard machine learning algorithms such as Gaussian Mixture Models, kNN and OCSVM. They find that AHMMAS performs better in terms of the area under the ROC curve and the F-measure.

The remainder of this paper proceeds as follows. In Section 2 we use market data to show the relationship between volume imbalance and the arrival of MOs and LOs. In Section 3 we introduce the model. In Section 4 we solve the optimisation problem of the investor and derive the optimal spoofing strategy. In Section 5 we employ simulations to illustrate the performance of the optimal strategy. We introduce the numerical scheme and prove the convergence results in Section 6. Finally, we conclude in Section 7 and collect proofs in Appendix.

\section{Limit order book and volume imbalance}

In this section we provide a measure of volume imbalance in the LOB and discuss its relationship with the arrival rates and volumes of MOs and LOs. We employ Nasdaq data for October 2017 to compute arrival rates and average sizes of MOs and LOs for 10 stocks. We remove the first and the last 30 minutes of each trading day to exclude the behaviour of the LOB during the opening and closing auctions of each trading day.

\subsection{Volume imbalance}

We define volume imbalance in the LOB at time $t$ as

$$
\rho_{t}=\frac{V_{t}^{b}-V_{t}^{a}}{V_{t}^{b}+V_{t}^{a}} \in(-1,1),
$$


where $V_{t}^{b}>0$ and $V_{t}^{a}>0$ are the volumes at time $t$ of LOs posted at the best bid and the best ask, respectively. Volume imbalance is a key quantity because it summarises the willingness of agents to buy or sell assets using LOs. Clearly, when $\rho_{t}$ is close to 1 there is strong buy pressure and when it is close to -1 there is strong sell pressure. Figure 1 shows the path of $\rho_{t}$ for stock INTC during a window of 2 minutes. Figure 2 shows the number of MOs (buy and sell) for different levels of volume imbalance for INTC during October 2017. We observe that as the absolute value of volume imbalance increases, the number of MOs increases.

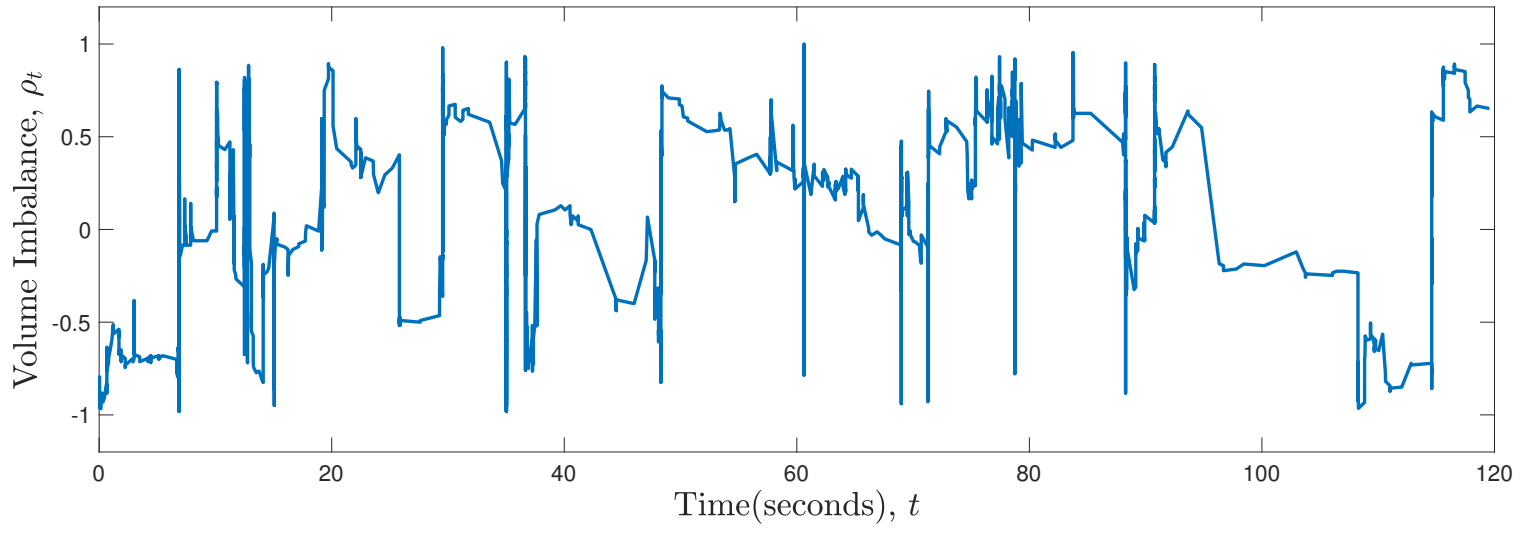

Figure 1: Volume imbalance $\rho_{t}$ of INTC from 10:00:00 to 10:02:00 on October 22017.

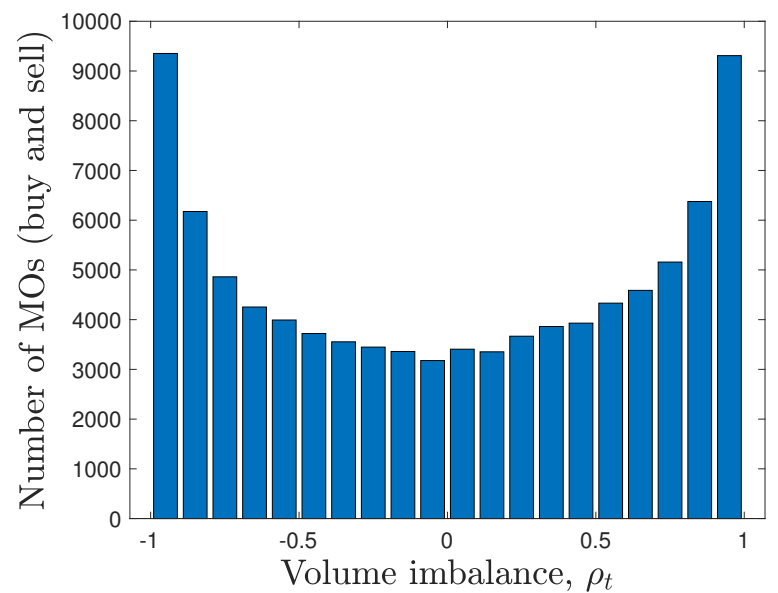

Figure 2: Number of MOs for various levels of volume imbalance $\rho_{t}$ of INTC. Data: Nasdaq October 2017.

Cartea et al. (2018) show that the volume imbalance measure (1) helps to predict the rate of incoming MOs and to predict the direction and magnitude of price movements that follow the arrival of a MO. We divide the range of the volume imbalance measure $(-1,1)$ into three subintervals. We refer to the interval $(1 / 3,1)$ as the buy-heavy regime, the interval $[-1 / 3,1 / 3]$ as the neutral regime, and the interval $(-1,-1 / 3)$ as the sell-heavy regime. When volume imbalance is buy-heavy (sell-heavy) and a MO arrives, there is a high probability that this MO is a buy (sell) order. Furthermore, immediately following a buy (sell) MO, the magnitude and sign of the change in the midprice is, on average, positive (negative) when volume imbalance is buy-heavy (sell-heavy). Table 1 shows the arrival rates of changes 
in the midprice conditioned on the volume imbalance regimes for 10 stocks in October 2017. For most stocks, the rate of midprice increase is highest in the buy-heavy regime and lowest in the sell-heavy regime, which indicates the predictive power of volume imbalance on price movements. For example, for the stock INTC in the buy-heavy regime, the arrival intensity of positive jumps in prices is 0.112 per second.

\begin{tabular}{crrr|rrr}
\hline \hline & \multicolumn{2}{c|}{$\begin{array}{c}\text { Arrival rates (per second) } \\
\text { Midprice increase }\end{array}$} & \multicolumn{3}{c}{$\begin{array}{c}\text { Arrival rates (per second) } \\
\text { Midprice decrease }\end{array}$} \\
\cline { 2 - 7 } & Buy-heavy & Neutral & Sell-heavy & Buy-heavy & Neutral & Sell-heavy \\
\hline \hline INTC & 0.112 & 0.407 & 0.044 & 0.048 & 0.412 & 0.096 \\
AAPL & 1.207 & 1.436 & 0.609 & 0.635 & 1.413 & 1.133 \\
BIDU & 0.543 & 0.339 & 0.389 & 0.417 & 0.343 & 0.525 \\
MSFT & 0.164 & 3.203 & 0.080 & 0.084 & 3.207 & 0.160 \\
AMZN & 0.861 & 0.659 & 0.607 & 0.610 & 0.624 & 0.793 \\
GOOG & 0.508 & 0.456 & 0.548 & 0.489 & 0.448 & 0.540 \\
NVDA & 1.724 & 1.302 & 1.016 & 1.027 & 1.081 & 1.897 \\
CSCO & 0.030 & 0.134 & 0.014 & 0.013 & 0.118 & 0.032 \\
\hline \hline
\end{tabular}

Table 1: Arrival rates of midprice increase/decrease (per second). Data: Nasdaq October 2017, with first and last 30 minutes of each trading day removed.

\subsection{Market order activity}

Table 2 shows the arrival rates (per second) of buy and sell MOs in each regime of volume imbalance for 10 stocks in October 2017. For most stocks, the arrival rate of buy MOs is highest in the buy-heavy regime and lowest in the sell-heavy regime. Similarly, for most stocks, the arrival rate of sell MOs is highest in the sell-heavy regime and lowest in the buy-heavy regime. When the LOB is buy-heavy (sell-heavy), the arrival rate of buy (sell) MOs is highest because some traders anticipate an increase (decrease) in prices, see Cartea et al. (2018). So, rather than posting LOs in the book, they send buy (sell) MOs to ensure they receive immediate execution at the best ask (bid) price 'before' prices increase (decrease).

\begin{tabular}{crrr|rrr}
\hline \hline & \multicolumn{2}{c|}{ Buy MO arrival rates (per second) } & \multicolumn{3}{c}{ Sell MO arrival rates (per second) } \\
\cline { 2 - 7 } & Buy-heavy & Neutral & Sell-heavy & Buy-heavy & Neutral & Sell-heavy \\
\hline \hline INTC & 0.270 & 0.051 & 0.047 & 0.040 & 0.052 & 0.312 \\
AAPL & 0.631 & 0.225 & 0.174 & 0.152 & 0.219 & 0.653 \\
BIDU & 0.110 & 0.076 & 0.135 & 0.168 & 0.090 & 0.124 \\
MSFT & 0.402 & 0.089 & 0.077 & 0.066 & 0.094 & 0.490 \\
AMZN & 0.196 & 0.144 & 0.206 & 0.237 & 0.147 & 0.171 \\
GOOG & 0.078 & 0.062 & 0.115 & 0.106 & 0.062 & 0.079 \\
NVDA & 0.299 & 0.215 & 0.273 & 0.277 & 0.215 & 0.300 \\
CSCO & 0.138 & 0.021 & 0.015 & 0.016 & 0.019 & 0.111 \\
\hline \hline
\end{tabular}

Table 2: Arrival rates of MOs (per second). Data: Nasdaq October 2017, with first and last 30 minutes of each trading day removed.

Table 3 shows the average volume of MOs in each regime of volume imbalance. The average volume of a buy MO in the buy-heavy regime is less than the average volume in the sell-heavy regime. This shows that although liquidity takers increase the rate at which they send buy MOs when volume imbalance is buy-heavy, the average size of the buy MOs is smaller than the average size of the sell MOs when the LOB is neutral or sell-heavy. We find similar results for sell MOs. The last column in the table shows the average daily volume of traded, buy and sell, MOs. 


\begin{tabular}{crrr|rrr|r}
\hline \hline & \multicolumn{2}{c|}{ Buy MO average volume } & \multicolumn{2}{c|}{ Sell MO average volume } & \multirow{2}{*}{$\begin{array}{c}\text { Avg. daily } \\
\text { volume }\end{array}$} \\
\cline { 2 - 6 } & Buy-heavy & Neutral & Sell-heavy & Buy-heavy & Neutral & Sell-heavy & $2,353,814$ \\
\hline \hline INTC & 414 & 904 & 734 & 703 & 872 & 392 & 2,35 \\
AAPL & 114 & 218 & 315 & 390 & 231 & 123 & $2,363,229$ \\
BIDU & 47 & 95 & 135 & 138 & 96 & 44 & 416,692 \\
MSFT & 232 & 449 & 456 & 480 & 456 & 230 & $2,090,697$ \\
AMZN & 25 & 59 & 100 & 95 & 60 & 24 & 464,922 \\
GOOG & 21 & 53 & 68 & 68 & 53 & 19 & 163,120 \\
NVDA & 53 & 109 & 242 & 236 & 111 & 50 & $1,303,358$ \\
CSCO & 562 & 927 & 684 & 813 & 1064 & 539 & $1,136,837$ \\
\hline \hline
\end{tabular}

Table 3: Average volume of MOs. Data: Nasdaq October 2017, with first and last 30 minutes of each trading day removed.

\subsection{Limit order activity}

Table 4 shows the arrival rates of LOs (per second) in each regime of volume imbalance. For most stocks, the arrival rate of buy LOs is highest in the buy-heavy regime and lowest in the sell-heavy regime. Similarly, for most stocks, the arrival rate of sell LOs is highest in the sell-heavy regime and lowest in the buy-heavy regime. These results are similar to those described above for the arrival rate of MOs, however the arrival rates of LOs are larger than the arrival rates of MOs.

\begin{tabular}{cccc|ccc}
\hline \hline & \multicolumn{2}{c|}{ Buy LO arrival rates (per second) } & \multicolumn{2}{c}{ Sell LO arrival rates (per second) } \\
\cline { 2 - 7 } & Buy-heavy & Neutral & Sell-heavy & Buy-heavy & Neutral & Sell-heavy \\
\hline \hline INTC & 5.00 & 2.50 & 2.21 & 1.98 & 2.48 & 5.36 \\
AAPL & 5.22 & 4.04 & 4.37 & 4.02 & 4.16 & 5.97 \\
BIDU & 0.43 & 0.34 & 0.42 & 0.40 & 0.30 & 0.38 \\
MSFT & 5.83 & 3.07 & 3.46 & 2.89 & 3.12 & 6.93 \\
AMZN & 0.62 & 0.53 & 0.47 & 0.49 & 0.52 & 0.54 \\
GOOG & 0.44 & 0.41 & 0.53 & 0.43 & 0.39 & 0.41 \\
NVDA & 2.39 & 2.35 & 2.28 & 1.21 & 1.20 & 1.19 \\
CSCO & 2.98 & 1.32 & 0.97 & 1.01 & 1.29 & 2.71 \\
\hline
\end{tabular}

Table 4: Arrival rates of LOs (per second). Data: Nasdaq October 2017, with first and last 30 minutes of each trading day removed.

Table 5 shows the average size of LOs in each regime of volume imbalance. For each stock, the average LO size remains approximately the same in the three regimes. The average size of the orders can be thought of as one lot of shares when we develop the mathematical framework below in Section 3 .

\begin{tabular}{crrr|rrr}
\hline & \multicolumn{2}{c|}{ Buy LO average volume } & \multicolumn{3}{c}{ Sell LO average volume } \\
\cline { 2 - 7 } & Buy-heavy & Neutral & Sell-heavy & Buy-heavy & Neutral & Sell-heavy \\
\hline \hline INTC & 320 & 313 & 327 & 347 & 319 & 310 \\
AAPL & 163 & 151 & 150 & 148 & 151 & 167 \\
BIDU & 72 & 75 & 59 & 65 & 80 & 78 \\
MSFT & 140 & 139 & 142 & 137 & 136 & 138 \\
AMZN & 59 & 58 & 47 & 49 & 56 & 55 \\
GOOG & 64 & 60 & 55 & 53 & 56 & 55 \\
NVDA & 56 & 57 & 55 & 79 & 82 & 81 \\
CSCO & 423 & 379 & 419 & 426 & 378 & 410 \\
\hline \hline
\end{tabular}

Table 5: Average volume of LOs. Data: Nasdaq October 2017, with first and last 30 minutes of each trading day removed. 


\subsection{Volumes at best prices and spoofing}

The previous two subsections showed that volume imbalance conveys important information about market activity of liquidity providers and takers. In most electronic markets it is almost costless to cancel or amend LOs, hence a trader can intentionally manipulate the volume imbalance in the LOB by posting LOs on one side of the LOB to take advantage of traders who use volume imbalance as one of the inputs in their trading strategies.

For example, suppose an investor wants to sell stocks. She could sell the stocks by sending MOs to the exchange, which guarantee immediate execution and pay the costs of crossing the spread plus other fees. Alternatively, although illegal, the investor can spoof the LOB. The trading strategy is based on the following two actions. (i) Post sell LOs at the best ask price in the LOB. (ii) Post spoof buy LOs on the best bid. The volumes of the spoof buy LOs must be large enough to tilt, momentarily, the LOB into the buy-heavy regime. Some traders will interpret this shift in regime as a signal of a likely increase in the price of the stock, so they send buy MOs that are likely to be filled by the spoofer's LOs resting on the sell side on the book.

Table 6 shows the time-weighted average volumes posted at the best bid and best ask prices in each regime of volume imbalance for the period October 2017 (first and last 30 minutes of each trading day removed). The information in the table provides a rough idea of the volume of spoof orders required to tilt the LOB. For example, to spoof the bid side of the book of INTC from sell-heavy to buy-heavy the investor must post spoof LOs that add up to approximately 10,154 shares.

\begin{tabular}{crrr|rrr}
\hline \hline & \multicolumn{2}{c|}{ Best bid average volume } & \multicolumn{3}{c}{ Best ask average volume } \\
\cline { 2 - 7 } & Buy-heavy & Neutral & Sell-heavy & Buy-heavy & Neutral & Sell-heavy \\
\hline \hline INTC & 6,165 & 3,376 & 1,513 & 1,660 & 3,313 & 5,833 \\
AAPL & 879 & 436 & 217 & 189 & 438 & 1,143 \\
BIDU & 323 & 162 & 47 & 68 & 160 & 271 \\
MSFT & 2,598 & 1,519 & 735 & 742 & 1,473 & 3,130 \\
AMZN & 230 & 115 & 26 & 27 & 113 & 225 \\
GOOG & 204 & 116 & 24 & 25 & 114 & 173 \\
NVDA & 535 & 177 & 76 & 81 & 178 & 570 \\
CSCO & 8,360 & 4,304 & 1,948 & 2,260 & 4,326 & 7,129 \\
\hline \hline
\end{tabular}

Table 6: Time-weighted average volume posted at the best bid and best ask prices. Data: Nasdaq October 2017, with first and last 30 minutes of each trading day removed.

\section{The model}

In this section we present a model for an investor who wants to liquidate a certain amount of inventory of a traded stock. Although spoofing is illegal, we assume that the investor is willing to spoof the LOB to improve the PnL from liquidating her inventory. We work on a completed filtered probability space $\left.\left(\Omega, \mathcal{F},(\mathcal{F})_{t \geq 0}\right), \mathbb{P}\right)$, where the filtration is the natural filtration generated by the collection of observable stochastic processes that we define below.

The finite state imbalance regime process without spoofing is $Z_{t} \in\{1,2,3\}$, which represents $\{b u y$ heavy, neutral, sell-heavy\} respectively, and we assume that $Z$ is a continuous-time Markov chain with 
constant generator matrix $G$.

The midprice of the asset is denoted by $S=\left(S_{t}\right)_{t \geq 0}$ and is given by

$$
S_{t}=S_{0}+\sigma\left(J_{t}^{+}-J_{t}^{-}\right)
$$

where $J^{ \pm}=\left(J_{t}^{ \pm}\right)_{t \geq 0}$ are conditionally independent doubly stochastic Poisson processes, with regimedependent arrival rates $\gamma^{ \pm}\left(Z_{t}\right)>0$ when there is no spoofing and $\sigma>0$ denotes the size of the tick in the LOB, which is assumed to be constant. The spread between the best bid and best ask prices is constant at $2 \Delta$ and we refer to $\Delta>0$ as the half-spread. One could consider a model where the spread is also stochastic, see Cartea et al. (2018). For simplicity, we assume the spread is constant at one tick, which is the case of the large-stick stocks such as the ones we study here.

\subsection{Liquidating shares without spoofing}

The investor wishes to sell $\mathfrak{N}>0$ shares before or by a fixed time horizon $T>0$. The investor can post sell LOs at the price $S_{t}+\Delta$, and wait for buy MOs from other market participants to trade with her LOs resting in the book. Every time a sell LO is filled, the investor receives $S_{t}+\Delta+\varepsilon_{L O}$, which is the best ask price in the LOB plus the liquidity making rebate $\varepsilon_{L O}>0$. We denote the total effective half-spread of a LO by $\Upsilon_{L O}:=\Delta+\varepsilon_{L O}$. Also, the investor can aggressively cross the spread by submitting sell MOs. We assume there is enough liquidity in the best bid of the LOB to fill the investor's sell MO. The investor's market sell order receives the cash $S-\Delta-\varepsilon_{M O}$ per share, which is the best bid price in the LOB minus the liquidity taking fee $\varepsilon_{M O} \geq 0$. We denote the total effective half-spread of a MO by $\Upsilon_{M O}:=\Delta+\varepsilon_{M O}$. The volume of the investor's sell LOs and the volume of the investor's sell MOs are one - e.g., one share or one lot of shares.

The arrival rate of MOs is assumed to be constant in each regime of volume imbalance. We denote by $\lambda^{+}\left(Z_{t}\right)$ the arrival rate of buy MOs, where $\lambda^{+}(1)>\lambda^{+}(2)>\lambda^{+}(3)$ - see for example Table 2. Finally, when a buy MO arrives, the sell LO of the investor is filled with probability $p_{\text {sell }}$, which we assume to be constant.

\subsection{Liquidating shares with spoof buy LOs}

In addition to the strategy described above, the investor can also include spoof orders as part of her trading strategy. That is, the investor employs a combination of: sell LOs at the best ask price, sell MOs, and spoof buy LOs at the best bid price $S_{t}-\Delta$ and recall that every time the spoof buy LO is filled, the investor receives a liquidity making rebate of $\varepsilon_{L O}$ per share. The volume of the spoof buy LOs must be large enough to tilt the book into the buy-heavy regime. This 'phantom liquidity' on the buy side of the LOB makes traders believe that volume imbalance is buy-heavy. This entices other market participants to post buy LOs (see Table 4) and the more impatient traders will send buy MOs in anticipation of an increase in prices, thus the arrival rate of buy MOs also increases (see Table 2). Consequently, the fill rate of the sell LOs posted by the investor increases, so the investor expects to liquidate her inventory of shares more quickly and at better prices. The sizes of the spoof buy LOs depend on the volume required to tilt the LOB into buy-heavy regime, see Table 6.

We denote the arrival rate of buy MOs in a LOB with spoof buy LOs by $\lambda^{s}$. We assume that the rate 
is constant and is equal to the arrival rate of buy MOs when the book is buy-heavy, i.e., $\lambda^{s}=\lambda^{+}(1)$. We note that if volume imbalance without spoofing is already in the buy-heavy regime, the investor does not need to spoof the LOB because the arrival rate of buy MOs is already highest.

When the investor spoofs the buy side of the LOB, the arrival rates of $J^{ \pm}$(i.e., arrival of price innovations) become $\gamma^{ \pm}(1)$ because the market believes that volume imbalance is buy-heavy. Therefore, market participants change their strategies in anticipation to an increase in the price of the asset. That is, some traders send buy MOs to purchase the asset and other traders reshuffle their LOs in the book. Both strategies are self-fulfilling because they exert an upward pressure in the price of the asset. This is an extreme example where the spoofer misleads market participants and the price of the asset deviates from its 'true' price - in an efficient market the price of the asset will eventually return to its true value.

The investor bears the risk that an incoming sell MO from another market participant hits her spoof buy LOs, which is against the investor's goal of selling shares in the stock. We denote by $\lambda^{-}$the arrival rate of sell MOs in the buy-heavy regime. When a sell MO arrives, $V$ shares in the spoof buy LOs of the investor are filled with probability $p_{\text {buy }}\left(Z_{t}\right)$. The value of $p_{\text {buy }}\left(Z_{t}\right)$ is determined by the volume of the spoof LOs, which itself depends on the imbalance regime of the LOB immediately before tilting the book with spoof orders. Clearly, the proportion of spoof LOs in the best bid queue is highest when the LOB was sell-heavy immediately before the investor sends the spoof orders. Thus, the probability that a spoof LO is filled is such that $p_{b u y}(2)<p_{\text {buy }}(3)$, which is generally borne out by the data (recall that regime 3 is when the book is sell-heavy). Finally, the investor mitigates the risk that her spoof buy LOs are filled by setting an upper bound, denoted by $\bar{Q}$ which is greater than $\mathfrak{N}$, on the inventory she is willing to hold.

\section{Investor's optimisation problem}

The control process $c=\left(c_{t}\right)_{t \geq 0}$ takes values in $\{0,1\}$, where $c_{t}=1$ denotes that the investor spoofs the $\mathrm{LOB}$ and $c_{t}=0$ represents no spoofing. The investor cannot spoof the book when her inventory is greater than $\bar{Q}-V$ because it might increase beyond the cap $\bar{Q}$ if the spoof LOs are filled (recall that the investor imposes the cap $\bar{Q}$ on the inventory holdings and $V$ represents the number of shares that every time the spoof buy LO is filled by. The investor does not need to spoof the LOB when volume imbalance is already buy-heavy, because the arrival rate of buy MOs is highest. We denote by $\mathcal{U}$ the set of admissible strategies $c$, consisting of $\mathcal{F}_{t}$-predictable control process $c \in\{0,1\}$, such that the inventory does not exceed the upper bound $\bar{Q}$, and $c_{t}=0$ when $Z_{t}=1$ because the book is already in the buy-heavy regime.

We represent by $N^{ \pm, c}=\left(N_{t}^{ \pm, c}\right)_{t \geq 0}$ the counting processes that keep track of the number of filled sell $(+)$ and buy $(-)$ LOs posted by the investor.

The investor also controls the times at which she sends sell MOs. These times are given by the impulse control $\boldsymbol{\tau}=\left(\tau_{1}, \tau_{2}, \tau_{3}, \ldots\right)$ and $M_{t}=\sum_{k=1}^{\infty} \mathbb{1}_{\left\{\tau_{k} \leq t\right\}}$ represents the counting process of the investor's sell MOs. We denote by $\mathcal{V}$ the set of admissible strategies $\tau$, which consists of increasing $\mathcal{F}_{t}$-stopping times less than $T$. 
Denote by $Q^{c, \boldsymbol{\tau}}=\left(Q_{t}^{c, \boldsymbol{\tau}}\right)_{t \geq 0}$ the investor's controlled inventory, which satisfies

$$
\mathrm{d} Q_{t}^{c, \boldsymbol{\tau}}=\mathrm{d} N_{t}^{-, c}-\mathrm{d} N_{t}^{+, c}-\mathrm{d} M_{t}
$$

and $X^{c, \boldsymbol{\tau}}=\left(X_{t}^{c, \boldsymbol{\tau}}\right)_{t \geq 0}$ represents the cash process with dynamics

$$
\mathrm{d} X_{t}^{c, \boldsymbol{\tau}}=\left(S_{t}^{c}+\Upsilon_{L O}\right) \mathrm{d} N_{t}^{+, c}-\left(S_{t}^{c}-\Upsilon_{L O}\right) \mathrm{d} N_{t}^{-, c}+\left(S_{t}^{c}-\Upsilon_{M O}\right) \mathrm{d} M_{t}
$$

The time $\tau_{\mathcal{S}}=\inf \left\{t: Q_{t}^{c, \tau}=0\right\}$ is when the investor sells all her shares and stops trading.

The investor's value function is

$$
\begin{array}{r}
H(t, x, S, q, Z)=\sup _{\left(c_{t}, \boldsymbol{\tau}\right) \in \mathcal{A}} \mathbb{E}_{t, x, S, q, Z}\left[\begin{array}{r}
X_{\tau_{\mathcal{S}} \wedge T}^{c, \boldsymbol{\tau}}+Q_{\tau_{\mathcal{S}} \wedge T}^{c, \boldsymbol{\tau}}\left(S_{\tau_{\mathcal{S}} \wedge T}^{c}-\Upsilon_{M O}-\alpha Q_{\mathcal{S}_{\mathcal{S}} \wedge T}^{c, \boldsymbol{\tau}}\right) \\
\left.-\phi_{q} \int_{t}^{\tau_{\mathcal{S}} \wedge T}\left(Q_{u}^{c, \boldsymbol{\tau}}\right)^{2} \mathrm{~d} u-\phi_{f} \int_{t}^{\tau_{\mathcal{S}} \wedge T} c_{u} \mathrm{~d} u\right]
\end{array}\right.
\end{array}
$$

where $\mathbb{E}_{t, x, S, q, Z}[\cdot]$ is the expectation operator conditional on $X_{t-}^{c, \boldsymbol{\tau}}=x, S_{t-}^{c}=S, Q_{t-}^{c, \boldsymbol{\tau}}=q$, and on the regime $Z$ of the volume imbalance. The set of admissible strategies is represented by $\mathcal{A}=\mathcal{U} \times \mathcal{V}$, which consists of $\mathcal{F}_{t}$-stopping times and $\mathcal{F}_{t}$-predictable control process $c \in\{0,1\}$, such that the inventory does not exceed the upper bound $\bar{Q}$, and $c_{t}=0$ when $Z_{t}=1$ because the book is already in the buy-heavy regime.

We give a brief interpretation of the terms in the value function. The term $X_{\tau_{\mathcal{S}} \wedge T}^{c, \tau}$ is the terminal cash held by the investor. The term $Q_{\tau_{\mathcal{S}} \wedge T}^{c, \tau}\left(S_{\tau_{\mathcal{S}} \wedge T}^{c}-\Upsilon_{M O}-\alpha Q_{\tau_{\mathcal{S}} \wedge T}^{c, \tau}\right)$ is the revenue from liquidating any remaining inventory using sell MOs at the terminal time. Here, $\alpha Q_{\tau_{\mathcal{S}} \wedge T}^{c, \tau}$ represents the cost of walking the LOB and the terminal inventory penalty parameter is a non-negative constant. The term, $\phi_{q} \int_{t}^{\tau_{\mathcal{S}} \wedge T}\left(Q_{u}^{c, \boldsymbol{\tau}}\right)^{2} \mathrm{~d} u$, is a running inventory penalty, where the non-negative constant $\phi_{q}$ is the running inventory penalty parameter, see Cartea and Jaimungal (2015b), Cartea et al. (2015), Guéant (2016). The last term, $\phi_{f} \int_{t}^{\tau_{\mathcal{S}} \wedge T} c_{u} \mathrm{~d} u$, represents the fine imposed by financial authorities if they detect spoofing and manipulation of prices, where the parameter $\phi_{f} \geq 0$ determines the severity of the fine.

By standard results, see Øksendal and Sulem (2007), the value function (5) is the unique viscosity 
solution of the Hamilton-Jacobi-Bellman quasi-variational inequality (HJBQVI)

$$
\begin{aligned}
& \max \left\{\partial_{t} H-\phi_{q} q^{2}\right. \\
& +\sup _{c \in \mathcal{U}}\left[c \left(-\phi_{f}+\gamma^{+}(1)(H(t, x, S+\sigma, q, Z)-H(t, x, S, q, Z))\right.\right. \\
& +\gamma^{-}(1)(H(t, x, S-\sigma, q, Z)-H(t, x, S, q, Z)) \\
& +\lambda^{s} p_{\text {sell }}\left(H\left(t, x+\left(S+\Upsilon_{L O}\right), S, q-1, Z\right)-H\right) \\
& \left.+\lambda^{-} p_{\text {buy }}(Z)\left(H\left(t, x-V\left(S-\Upsilon_{L O}\right), S, q+V, Z\right)-H\right)\right) \\
& +(1-c)\left(\gamma^{+}(Z)(H(t, x, S+\sigma, q, Z)-H(t, x, S, q, Z))\right. \\
& +\gamma^{-}(Z)(H(t, x, S-\sigma, q, Z)-H(t, x, S, q, Z)) \\
& \left.\left.+\lambda^{+}(Z) p_{\text {sell }}\left(H\left(t, x+\left(S+\Upsilon_{L O}\right), S, q-1, Z\right)-H\right)\right)\right] \\
& +\sum_{K}(H(t, x, S, q, K)-H(t, x, S, q, Z)) G_{Z, K}, \\
& \left.H\left(t, x+\left(S-\Upsilon_{M O}\right), S, q-1, Z\right)-H\right\}=0
\end{aligned}
$$

with terminal and boundary conditions

$$
H(T, x, S, q, Z)=x+q\left(S-\Upsilon_{M O}-\alpha q\right) \quad \text { and } \quad H(t, x, S, 0, Z)=x,
$$

respectively. For simplicity, we suppress the arguments of $H(t, x, S, q, Z)$ and write $H$; we do the same for other functions we define later.

If $q<\bar{Q}$ and

$$
\begin{aligned}
& -\phi_{f}+\gamma^{+}(1)(H(t, x, S+\sigma, q, Z)-H)+\gamma^{-}(1)(H(t, x, S-\sigma, q, Z)-H) \\
& +\lambda^{s} p_{\text {sell }}\left(H\left(t, x+\left(S+\Upsilon_{L O}\right), S, q-1, Z\right)-H\right) \\
& +\lambda^{-} p_{\text {buy }}(Z)\left(H\left(t, x-V\left(S-\Upsilon_{L O}\right), S, q+V, Z\right)-H\right) \\
> & \gamma^{+}(Z)(H(t, x, S+\sigma, q, Z)-H)+\gamma^{-}(Z)(H(t, x, S-\sigma, q, Z)-H) \\
& +\lambda^{+}(Z) p_{\text {sell }}\left(H\left(t, x+\left(S+\Upsilon_{L O}\right), S, q-1, Z\right)-H\right),
\end{aligned}
$$

then the optimal control process in feedback form is

$$
c^{*}(t, x, S, q, Z)=1 .
$$


Otherwise, $c^{*}(t, x, S, q, Z)=0$.

The left-hand side of the inequality above shows the expected change in the value function when the investor rests a sell LO in the book and spoofs the buy side of the book. Specifically, (7) consists of five terms. The first term represents the fine imposed by financial authorities if they detect spoofing and manipulation of prices. The second and the third terms represent the change in the value function when the midprice moves. The intensities $\gamma^{ \pm}$are those in the buy-heavy region because the investor spoofs the LOB into the buy-heavy regime. The fourth term represents the change in the value function when a sell LO is filled (with probability $\lambda^{s} p_{\text {sell }}$ ). The fifth term is the change in the value function when the investor's spoof buy LOs are filled (with probability $\lambda^{-} p_{\text {buy }}(Z)$ ). The right-hand side of the inequality, i.e., (8), consists of three parts. The first two terms represent the change in the value function when the midprice moves without spoofing. The third term is the change in the value function when the investor does not spoof the buy side of the book, so the sell LOs are filled with probability $\lambda^{+}(Z) p_{\text {sell }}$.

The optimal stopping times $\tau^{*}$ when the investor submits sell MOs satisfies

$$
\tau_{k}^{*}=\inf \left\{t>\tau_{k-1}^{*}, H\left(t, x+\left(S-\Upsilon_{M O}\right), S, q-1, Z\right)-H=0\right\}, \quad \text { for } k>1,
$$

and

$$
\tau_{1}^{*}=\inf \left\{t>0, H\left(t, x+\left(S-\Upsilon_{M O}\right), S, q-1, Z\right)-H=0\right\},
$$

i.e., the investor executes a $\mathrm{MO}$ when the change in the value function is zero.

Substitute ansatz $H(t, x, S, q, Z)=x+q S+\widetilde{h}(t, q, Z)$ in (6) to obtain

$$
\begin{aligned}
& \max \left\{\partial_{t} \widetilde{h}-\phi_{q} q^{2}\right. \\
& +\sup _{c \in \mathcal{U}}\left[c \left(-\phi_{f}+\left(\gamma^{+}(1)-\gamma^{-}(1)\right) \sigma q+\lambda^{s} p_{\text {sell }}\left(\Upsilon_{L O}+\widetilde{h}(t, q-1, Z)-\widetilde{h}\right)\right.\right. \\
& \left.+\lambda^{-} p_{\text {buy }}(Z)\left(V \Upsilon_{L O}+\widetilde{h}(t, q+V, Z)-\widetilde{h}\right)\right) \\
& \left.+(1-c)\left(\left(\gamma^{+}(Z)-\gamma^{-}(Z)\right) \sigma q+\lambda^{+}(Z) p_{\text {sell }}\left(\Upsilon_{L O}+\widetilde{h}(t, q-1, Z)-\widetilde{h}\right)\right)\right] \\
& \quad+\sum_{K \neq Z}(\widetilde{h}(t, q, K)-\widetilde{h}(t, q, Z)) G G_{Z, K}, \\
& \left.-\Upsilon_{M O}+\widetilde{h}(t, q-1, Z)-\widetilde{h}\right\}=0,
\end{aligned}
$$

with terminal and boundary conditions

$$
\widetilde{h}(T, q, Z)=q\left(-\Upsilon_{M O}-\alpha q\right) \quad \text { and } \quad \widetilde{h}(t, 0, Z)=0,
$$

respectively. 
Now, if $q<\bar{Q}$ and

$$
\begin{aligned}
& -\phi_{f}+\left(\gamma^{+}(1)-\gamma^{-}(1)\right) \sigma q \\
& +\lambda^{s} p_{\text {sell }}\left(\Upsilon_{L O}+\widetilde{h}(t, q-1, Z)-\widetilde{h}\right)+\lambda^{-} p_{\text {buy }}(Z)\left(V \Upsilon_{L O}+\widetilde{h}(t, q+V, Z)-\widetilde{h}\right) \\
& >\left(\gamma^{+}(Z)-\gamma^{-}(Z)\right) \sigma q+\lambda^{+}(Z) p_{\text {sell }}\left(\Upsilon_{L O}+\widetilde{h}(t, q-1, Z)-\widetilde{h}\right),
\end{aligned}
$$

the feedback control becomes

$$
c^{*}(t, q, Z)=1
$$

Otherwise, $c^{*}(t, q, Z)=0$.

The investor submits sell MOs at the stopping times $\tau^{*}$ such that

$$
\tau_{k}^{*}=\inf \left\{t>\tau_{k-1}^{*}, h(t, q-1, Z)-h=\Upsilon_{M O}\right\}, \quad \text { for } k>1,
$$

and

$$
\tau_{1}^{*}=\inf \left\{t>0, h(t, q-1, Z)-h=\Upsilon_{M O}\right\}
$$

Theorem 1. (Verification) Let $\widetilde{h}$ be a solution to (12) and define a candidate solution $\tilde{H}=x+q S+$ $\widetilde{h}(t, q, Z)$. Then $\tilde{H}$ equals the value function as defined in (5).

Proof. For a proof please see Appendix.

\subsection{Trading in lots of shares}

In our model setup, we assume the volume of sell LOs and buy MOs is one share and the volume that the spoof buy LOs is filled by is $V$ shares. It is straightforward to scale the size of the investor's orders by a factor $\psi>0$ and show that the value function of the investor scales linearly in $\psi$ if the running penalty and terminal inventory penalty parameters also scale with $\psi$. First, note that if the investor's buy MO and sell LO are of size $\psi$, then the cash she obtains per filled LO is $\hat{S}^{c}+\hat{\Upsilon}_{L O}$, where $\hat{S}^{c}=\psi S^{c}$ and $\hat{\Upsilon}_{L O}=\psi \Upsilon_{L O}$. Similarly, the price received for a sell MO of size $\psi$ is $\hat{S}^{c}-\hat{\Upsilon}_{M O}$, where $\hat{\Upsilon}_{M O}=\psi \Upsilon_{M O}$

Therefore, when the investor trades in lots of size $\psi$, her value function is

$$
\begin{aligned}
& \hat{H}(t, \hat{x}, \hat{S}, q, Z)=\sup _{\left(c_{t}, \boldsymbol{\tau}\right) \in \mathcal{A}} \mathbb{E}_{t, \hat{x}, \hat{S}, q, Z}\left[\hat{X}_{\tau_{\mathcal{S}}^{c} \wedge T}^{c, \boldsymbol{\tau}}+Q_{\tau_{\mathcal{S}} \wedge T}^{c, \boldsymbol{\tau}}\left(\hat{S}_{\tau_{\mathcal{S}} \wedge T}^{c}-\hat{\Upsilon}_{M O}-\hat{\alpha} Q_{\tau_{\mathcal{S}} \wedge T}^{c, \boldsymbol{\tau}}\right)\right. \\
& \left.-\hat{\phi}_{q} \int_{t}^{\tau_{\mathcal{S}} \wedge T}\left(Q_{u}^{c, \boldsymbol{\tau}}\right)^{2} \mathrm{~d} u-\hat{\phi}_{f} \int_{t}^{\tau_{\mathcal{S}} \wedge T} c_{u} \mathrm{~d} u\right]
\end{aligned}
$$

where $\hat{X}^{c, \tau}$ denotes the cash obtained from trading in lots of size $\psi$, and (with a slight abuse of notation) the units of inventory $Q^{c, \tau}$ and of the volume $V$ (lots of shares filled when a spoof buy LO is hit), and 
the counting processes for filled LOs and executed MOs are in lots of shares of size $\psi$.

If we assume $\hat{\phi}_{q}=\psi \phi_{q}, \hat{\phi}_{f}=\psi \phi_{f}$, and $\hat{\alpha}=\psi \alpha$ it is easy to see that $\hat{H}(t, \hat{x}, \hat{S}, q, Z)=\psi H(t, x, S, q, Z)$. Thus, the optimal strategies of the original problem and the scaled problem are the same, and for each path of the strategy, terminal cash is $\hat{X}_{\tau_{\mathcal{S}} \wedge T}^{c, \tau}=\psi X_{\tau_{\mathcal{S}} \wedge T}^{c, \tau}$.

\subsection{Optimal trading strategy}

We use Nasdaq data to estimate model parameters for the stock INTC in October 2017 — some parameter estimates are in Section 2, As in the empirical analysis above, we remove the first and last 30 minutes of each day to exclude the behaviour of the LOB during the opening and closing auctions of each trading day. We use the methodology in Cartea et al. (2018) to calculate the maximum likelihood estimates of the parameters. Recall that $\lambda^{-}$is the arrival rate of sell MOs when the volume imbalance is buy-heavy, $\lambda^{+}\left(Z_{t}\right)$ is the arrival rate of buy MOs when the investor does not spoof the LOB, $\lambda^{s}$ is the arrival rate of buy MOs when the investor spoofs the book, i.e., the investor tilts the book with buy LOs into the buy-heavy, and we assumed that $\lambda^{s}=\lambda^{+}(1)$. The estimate of the arrival rates (per second) are:

$$
\hat{\lambda}^{-}=0.0395, \quad \hat{\lambda}^{s}=\hat{\lambda}^{+}(1)=0.2698, \quad \hat{\lambda}^{+}(2)=0.0514, \quad \hat{\lambda}^{+}(3)=0.0469 .
$$

To ensure the model does not contain any long term speculation based on a non-zero drift in the midprice, we impose the symmetry constraints, as in Cartea et al. (2018), on the arrival rates of the innovations in the midprice of the asset and the generator matrix of the volume imbalance regime process $Z$. The estimates of the arrival rates of the innovations in the midprice of the asset (per second) and the generator matrix are, respectively, given by

$$
\hat{\gamma}^{+}=(0.1985,0.8195,0.0961), \quad \hat{\gamma}^{-}=(0.0961,0.8195,0.1985),
$$

and

$$
\hat{G}=\left[\begin{array}{rrr}
-0.8644 & 0.7642 & 0.1002 \\
0.3634 & -0.7268 & 0.3634 \\
0.1002 & 0.7642 & -0.8644
\end{array}\right]
$$

Other model parameters are (see e.g., Cartea and Jaimungal (2015a) and Foucault (2012)):

$$
\begin{gathered}
\Delta=0.005, \quad \varepsilon_{M O}=0.003, \quad \varepsilon_{L O}=0.002, \quad \alpha=0 \\
\mathfrak{N}=30 \text { shares }, \quad \bar{Q}=40 \text { shares }, \quad T=300 \text { seconds. }
\end{gathered}
$$

Figure 3 shows the optimal spoof strategy as a function of the inventory and the time remaining to expiry. The black area, denoted by LO, represents the region where the investor only posts sell LOs and does not spoof the book. The white area, denoted by Spoof, represents the region where the investor spoofs the book and also posts sell LOs, and the grey area, denoted by MO, represents the 
region where the investor executes sell MOs.

When market participants believe volume imbalance genuinely reflects demand and supply of the asset, and there is a potential fine for spoofing (i.e., $\phi_{f}=4.5 \times 10^{-3}$ ), the optimal strategy for the agent is to spoof the LOB when the book is sell-heavy and neutral; the exception in these regimes is when the strategy is near expiry or the inventory is very high. The agent does not spoof when the inventory level is very low because the risk of a fine outweighs the benefit of spoofing. Recall that the investor does not spoof when volume imbalance is already buy-heavy $(Z=1)$ because the arrival rate of buy MOs is highest and the price of the asset is expected to increase.
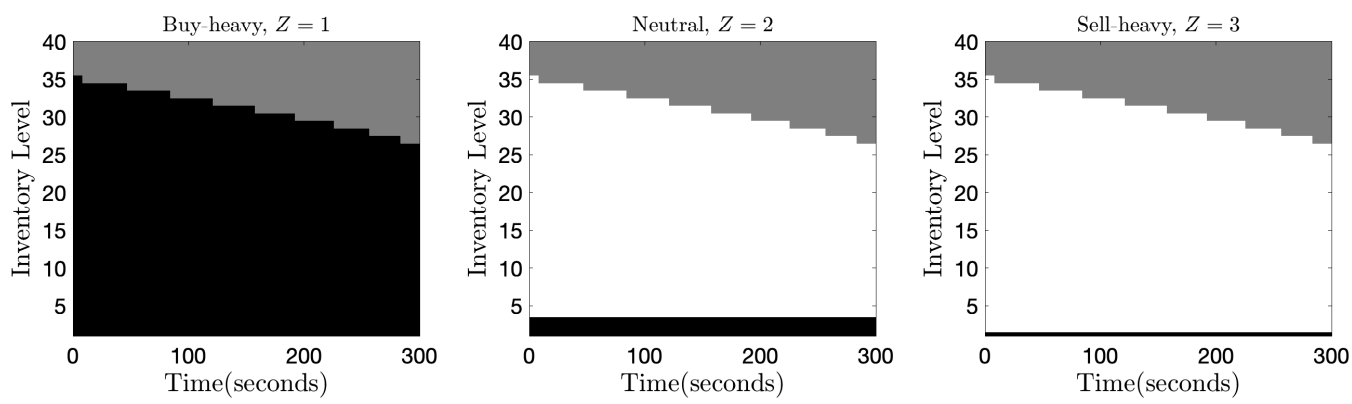

Figure 3: Optimal strategies for the investor. Parameter values as listed above and $\phi_{q}=2 \times 10^{-5}, \phi_{f}=4.5 \times 10^{-3}, p_{\text {sell }}=$ $0.3, p_{\text {buy }}=(0,0.5,0.6), V=2$. Black: investor only posts sell LOs and does not spoof the book. White: investor spoofs the book and also posts sell LOs. Grey: investor executes sell MOs.

\section{Simulation and performance of strategy}

In this section we employ simulations to analyse the performance of the optimal spoofing strategy. The initial midprice is $S_{0}=99.995$ and the tick size is $\sigma=0.01$, and the best bid and best ask prices are 99.99 and 100.00, respectively. We assume that market participants believe the information conveyed by the LOB, so the spoofer benefits from an increase in the arrival rate of sell MOs and from price manipulation - recall that in the buy-heavy the midprice of the asset increases on average.

\subsection{PnL and price manipulation}

We run 10,000 simulations to show the performance of the optimal strategy as a function of the fine parameter $\phi_{f}$. At time $T$ the investor's PnL is

$$
\operatorname{PnL}=X_{T}+Q_{T}\left(S_{T}-\Upsilon_{M O}-\alpha Q_{T}\right)
$$

where the units of inventory, LOs, and MOs is one lot of one share. As discussed in subsection 4.1. one can rescale the size of the LOs and MOs and the results derived here for lots of one share are easily reinterpreted. For example, the investor could trade lots of 300 shares in INTC, which is approximately the average size of the LOs posted on the book of INTC, see Table 5 - below we return to this particular example of lots of shares.

Table 7 shows statistics for the number of filled LOs and executed MOs when $\phi_{q}=10^{-5}, p_{\text {buy }}=$ $(0,0.5,0.6)$, and $V=2$. The top panel of the table reports the case where the investor does not spoof the LOB. The other three panels assume that the fine parameter $\phi_{f}$ takes on the values $\left\{4 \times 10^{-2}, 2.7 \times\right.$ 
$\left.10^{-2}, 0\right\}$ respectively. When the fine parameter $\phi_{f}$ is $4 \times 10^{-2}$ the expected penalty from spoofing is so high that is optimal for the investor not to spoof the LOB - compare the results of this case with those of no-spoof to observe that they are the same.

On the other hand, when the fine is zero, the results in the bottom panel show that the benefits from spoofing are considerable. On average, the investor sells more shares using LOs and fewer shares using MOs than the strategy without spoof LOs. The strategy fills an average of 9.5 spoof buy LOs, which are unwound (on average) with sell LOs. Although the spoof strategy bears the risk of fills on the 'wrong' side of the LOB, these inadvertently filled buy LOs help to increase the average PnL of the strategy. The mean PnL the investor obtains when there is no fine from spoofing is $\$ 12.13$ (which is approximately $\$ 0.40$ per share) greater than the mean $\mathrm{PnL}$ of the strategy without spoofing or when the fine parameter $\phi_{f}$ is $4 \times 10^{-2}$. This difference is approximately 1,213 times the quoted spread of INTC in the Nasdaq exchange. This example is extreme because the fine for spoofing is zero, so the investor spoofs the LOB for most of the trading horizon.

The PnL of the spoofing strategy is higher because: (i) the investor employs fewer MOs to draw the inventory to zero and also receives revenue from roundtrip trades, i.e., earns the spread (between the best ask and best bid prices) and the liquidity making rebate, when filled spoof buy LOs are unwound with sell LOs. (ii) The midprice trends upward when the book is buy-heavy. Thus, as time evolves, the spoofer obtains, on average, better prices for selling the asset.

Observe that the standard deviation of the PnL of the strategy with spoof LOs is higher than the standard deviation of the $\mathrm{PnL}$ when the investor does not spoof the book. We explain the intuition of this result. First, with our choice of inventory penalty parameter $\phi_{q}$, the no-spoof strategy liquidates the inventory, on average, with more sell MOs than sell LOs. On the other hand, the spoof strategy relies, on average, on more LOs and fewer MOs - thus, the PnL of the spoof strategy exhibits more variability than the PnL of the no-spoof strategy. Second, the liquidation programme with spoofing takes longer (mean time: 284.243 seconds in the case of zero fine) than the time it takes to liquidate the position (mean time: 190.035 seconds) without spoofing because the spoof strategy employs few MOs and many LOs. The execution time is the time when the inventory level first hits zero if it is before $T=300$ seconds, or 300 seconds if there is still inventory remaining at $T$.

Finally, note that the standard deviation of the PnL of the spoof case with $\phi_{f}=2.7 \times 10^{-2}$ is higher than the standard deviation of the PnL of a spoof strategy with zero fine. This result is explained by the volatility of the midprice, which is affected by the spoof strategy. In the buy-heavy regime the volatility of the midprice is lower than the volatility of the midprice when the LOB could be in any volume imbalance regime. When there is no fine for spoofing the book, the strategy tilts the book into the buy-heavy regime for most of the trading horizon, thus the volatility of the midprice is lower when $\phi_{f}=0$; we return to this below when we discuss price manipulation. 


\begin{tabular}{|c|c|c|c|c|c|c|c|c|c|c|}
\hline & & \multirow[b]{2}{*}{ mean } & \multirow[b]{2}{*}{ std } & \multicolumn{5}{|c|}{ Quantiles } & \multicolumn{2}{|c|}{$\mathrm{PnL}$} \\
\hline & & & & 0.01 & 0.25 & 0.50 & 0.75 & 0.99 & mean & std \\
\hline \multirow{2}{*}{ No Spoof } & filled sell LOs & 5.897 & 0.754 & 3 & 6 & 6 & 6 & 8 & \multirow{2}{*}{$2,999.688$} & \multirow{2}{*}{0.561} \\
\hline & executed sell MOs & 24.103 & 0.754 & 22 & 24 & 24 & 24 & 27 & & \\
\hline \multirow{2}{*}{\multicolumn{2}{|c|}{$\begin{array}{c}\text { Spoof } \\
\phi_{f}\end{array}$}} & & & \multicolumn{5}{|c|}{ Quantiles } & \multicolumn{2}{|c|}{ PnL } \\
\hline & & mean & std & 0.01 & 0.25 & 0.50 & 0.75 & 0.99 & mean & std \\
\hline \multirow{3}{*}{$4 \times 10^{-2}$} & filled sell LOs & 5.897 & 0.754 & 3 & 6 & 6 & 6 & 8 & \multirow{3}{*}{$2,999.688$} & \multirow{3}{*}{0.561} \\
\hline & executed sell MOs & 24.103 & 0.754 & 22 & 24 & 24 & 24 & 27 & & \\
\hline & filled buy LOs & 0 & 0 & 0 & 0 & 0 & 0 & 0 & & \\
\hline \multirow{3}{*}{$2.7 \times 10^{-2}$} & filled sell LOs & 15.654 & 2.924 & 10 & 14 & 15 & 17 & 24 & \multirow{3}{*}{$3,006.759$} & \multirow{3}{*}{4.153} \\
\hline & executed sell MOs & 18.823 & 3.458 & 13 & 16 & 18 & 21 & 30 & & \\
\hline & filled buy LOs & 4.477 & 4.476 & 0 & 2 & 4 & 6 & 20 & & \\
\hline \multirow{3}{*}{0} & filled sell LOs & 24.290 & 4.771 & 14 & 21 & 24 & 27 & 36 & \multirow{3}{*}{$3,011.818$} & \multirow{3}{*}{2.717} \\
\hline & executed sell MOs & 15.262 & 6.481 & 0 & 11 & 15 & 20 & 31 & & \\
\hline & filled buy LOs & 9.553 & 4.411 & 0 & 6 & 10 & 12 & 22 & & \\
\hline
\end{tabular}

Table 7: Each order is for one share of INTC. Parameters: $\phi_{q}=10^{-5}, p_{\text {sell }}=0.3, p_{\text {buy }}=(0,0.5,0.6), V=2, \mathfrak{N}=30$. Mean execution time in seconds: i) No Spoofing: 190.035 ; ii) Spoofing: $\phi_{f}=4 \times 10^{-2}: 190.035, \phi_{f}=2.7 \times 10^{-2}: 284.243$, $\phi_{f}=0: 299.638$.

Figure 4 shows heatmaps of the evolution of the price of the asset when the investor spoofs and does not spoof the LOB. Clearly, when there is spoofing, and market participants trust that volume imbalance is informative about the demand and supply of the asset, the midprice will exhibit an upward trend (see left panel of figure). Thus, the mean midprice at maturity is highest when the investor spoofs the LOB because the drift of the midprice is positive in this buy-heavy regime. Also, when the book is spoofed into the buy-heavy regime for a large proportion of the trading horizon, the midprice will exhibit lower volatility than when the LOB could be in any volume imbalance regime as is the case with no spoofing.

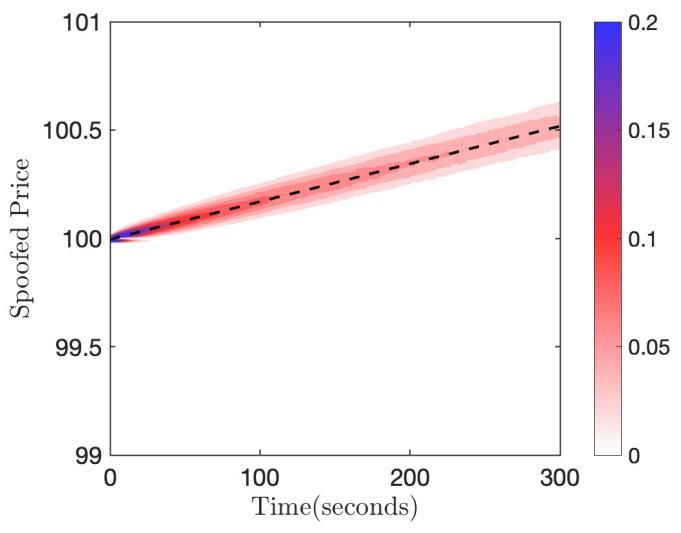

(a) $\operatorname{Mean}\left(S_{T}\right)=100.518$ and $\operatorname{Std}\left(S_{T}\right)=0.081$

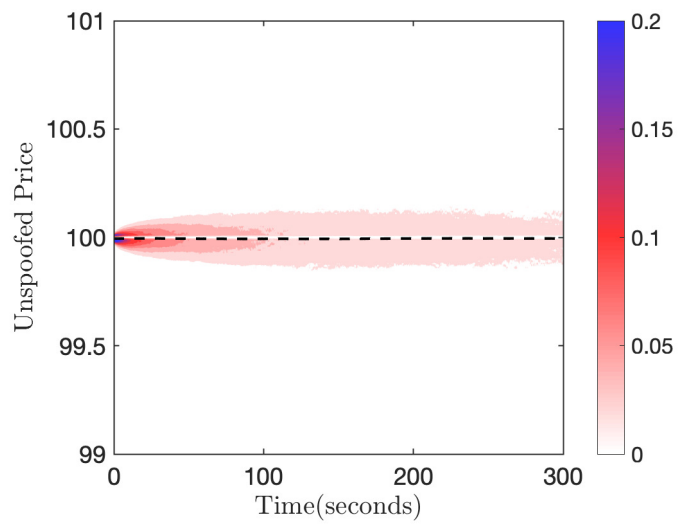

(b) $\operatorname{Mean}\left(S_{T}\right)=99.995$ and $\operatorname{Std}\left(S_{T}\right)=0.172$

Figure 4: Heatmaps of the evolution of the midprice. Parameters: $\phi_{q}=10^{-10}, \phi_{f}=0, p_{\text {sell }}=0.3, p_{\text {buy }}=$ $(0,0.5,0.6), V=2, \mathfrak{N}=30$.

Finally, for the examples discussed in Table 7, if we assume each lot consists of 300 shares, which is approximately the average volume of a LO posted at the best bid in INTC's book (see Table 5), the 
improvement in the mean PnL with spoofing compared with no spoofing is approximately $1,213 \times 300$ times the quoted spread in INTC. That is, the investor liquidates 9,000 shares (with scaled penalty parameters $\hat{\alpha}=300 \alpha, \hat{\phi}_{q}=300 \phi_{q}$, and $\left.\hat{\phi}_{f}=300 \phi_{f}\right)$ and MOs and LOs are for lots of 300 shares instead of lots of one share.

\subsection{Tradeoff: mean and standard deviation of PnL}

In this section we discuss the mean and the standard deviation of various performance measures that employ the following inputs: the PnL obtained by the investor from selling shares with and without spoofing and a market benchmark.

We introduce three measures of relative performance. The first is

$$
\mathrm{rPnL}_{1}=\mathrm{PnL}-\mathfrak{N}\left(S_{0}-\Upsilon_{M O}\right)
$$

where PnL is given by 16 .

The two other measures of relative performance are:

$$
\mathrm{rPnL}_{i}=\frac{\operatorname{PnL}_{- \text {benchmark }_{i}}}{\text { benchmark }_{i}} \times 10^{4}, \quad i=2,3,
$$

where benchmark ${ }_{2}=\mathfrak{N}\left(S_{0}-\Upsilon_{M O}\right)$ and benchmark $3=\mathrm{PnL}^{T W A P}$. Here, $\mathrm{PnL}^{T W A P}$ denotes the revenue obtained from a time-weighted-average-price strategy known as TWAP, i.e., the investor liquidates shares at the constant speed $\mathfrak{N} / T$. The measure of performance (18) represents the extra PnL of the spoof strategy relative to a benchmark in basis points.

We employ 10,000 simulations to compute the mean and the standard deviation of $\mathrm{rPnL}_{i}$ for $i=1,2,3$. The fine parameter $\phi_{f}$ takes values in the interval $\left[e^{-6}, e^{-2}\right]$, which is approximately $\left[2.5 \times 10^{-3}, 1.3 \times\right.$ $\left.10^{-1}\right]$. Other model parameters are $\phi_{q}=10^{-10}, p_{\text {sell }}=0.3, p_{\text {buy }}=(0,0.5,0.6), V=2, p_{\text {sell }}=0.3$.

Figure 5 depicts Mean $\left(\mathrm{rPnL}_{i}\right) / \operatorname{Std}\left(\mathrm{rPnL}_{i}\right)$, where the $x$-axis is in units of $\log \phi_{f}$. As expected, the difference between the performance of the spoof and that of the no-spoof strategies is highest when the fine for spoofing is smallest. As the fine for spoofing increases, the strategy relies on fewer spoof buy LOs because the expected fine outweighs the benefits from spoofing.

From panels (b) and (c) of Figure 5, we notice that for the TWAP benchmark, the difference in Mean $(\mathrm{rPnL}) / \mathrm{Std}(\mathrm{rPnL})$ between spoof and no-spoof, is smaller than when the benchmark is $\mathfrak{N}\left(S_{0}-\right.$ $\left.\Upsilon_{M O}\right)$. This shows, for our choice of parameters, that TWAP outperforms $\mathfrak{N}\left(S_{0}-\Upsilon_{M O}\right)$ and therefore the increase in Mean $(\mathrm{rPnL}) / \operatorname{Std}(\mathrm{rPnL})$ (panel (c)) is less significant than the case with $\mathfrak{N}\left(S_{0}-\Upsilon_{M O}\right)$ as benchmark (panel (b)). 


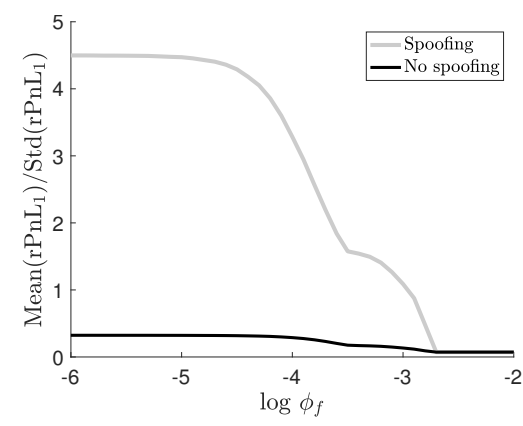

(a) $\mathrm{rPnL}_{1}=\operatorname{PnL}-\mathfrak{N}\left(S_{0}-\Upsilon_{M O}\right)$

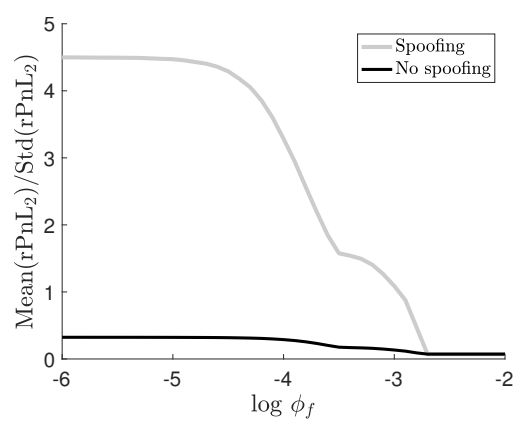

(b) benchmark $2: \mathfrak{N}\left(S_{0}-\Upsilon_{M O}\right)$

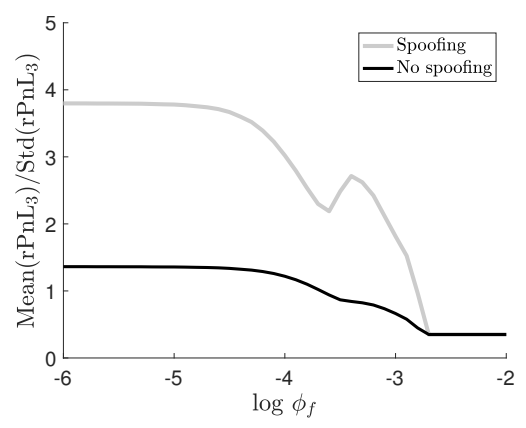

(c) benchmark $3: \mathrm{PnL}^{T W A P}$

Figure 5: Performance of strategy for $\phi_{f}$ in the range $\left[e^{-6}, e^{-2}\right]$. Other parameters: $\phi_{q}=10^{-10}, p_{\text {sell }}=0.3, p_{\text {buy }}=$ $(0,0.5,0.6), V=2, p_{\text {sell }}=0.3$.

\section{Numerical scheme}

In this section we describe the numerical scheme we employ to solve $(12)$. Let $\mathbb{T}_{\delta t}$ be the uniform grid on $[0, T]$ with step size $\delta t>0$. For any function $\varphi:[0, T] \times([0, \bar{Q}] \cap \mathbb{Z}) \times\{1,2,3\} \rightarrow \mathbb{R}$ define the operator

$$
\mathcal{S}^{\delta t}(t, q, Z, \varphi)=\max \left[\mathcal{T}^{\delta t}(t, q, Z, \varphi), \mathcal{M}^{\delta t}(t, q, Z, \varphi)\right]
$$

where

$$
\begin{aligned}
& \mathcal{T}^{\delta t}(t, q, Z, \varphi) \\
& =\varphi+\delta t\left[-\phi_{q} q^{2}\right. \\
& +\sup _{c \in \mathcal{U}}\left[c \left(-\phi_{f}+\left(\gamma^{+}(1)-\gamma^{-}(1)\right) \sigma q+\lambda^{s} p_{\text {sell }}\left(\Upsilon_{L O}+\varphi(t, q-1, Z)-(1+\kappa) \varphi\right)\right.\right. \\
& \left.\quad+\lambda^{-} p_{\text {buy }}(Z)\left(V \Upsilon_{L O}+\varphi(t, q+V, Z)-(1+\kappa) \varphi\right)\right) \\
& \left.\quad+(1-c)\left(\left(\gamma^{+}(Z)-\gamma^{-}(Z)\right) \sigma q+\lambda^{+}(Z) p_{\text {sell }}\left(\Upsilon_{L O}+\varphi(t, q-1, Z)-(1+\kappa) \varphi\right)\right)\right] \\
& \left.\quad+\sum_{K \neq Z}(\varphi(t, q, K)-(1+\kappa) \varphi(t, q, Z)) G_{Z, K}\right],
\end{aligned}
$$

and

$$
\mathcal{M}^{\delta t}(t, q, Z, \varphi)=\frac{\varphi(t, q-1, Z)-\Upsilon_{M O}}{1+\kappa}
$$

where $\kappa \downarrow 0$ is a robustness parameter. 
Define the numerical solution $h^{\delta t}: \mathbb{T}_{\delta t} \times([0, \bar{Q}] \cap \mathbb{Z}) \times\{1,2,3\} \rightarrow \mathbb{R}$ as follows:

$$
\begin{cases}h^{\delta t}(T, q, Z) & =-q\left(\Upsilon_{M O}+\alpha q\right) \\ h^{\delta t}(t, 0, Z) & =0 \\ h^{\delta t}(k \delta t, q, Z) & =\mathcal{S}^{\delta t}\left(t, q, Z, h^{\delta t}((k+1) \delta t, q, Z)\right) .\end{cases}
$$

We employ the explicit scheme backwards in time from $T$.

Next we prove the convergence of (22). We first prove monotonicity, stability, and consistency properties (Propositions 1, 2, and 3 respectively) of $\mathcal{S}^{\delta t}(t, q, Z, \varphi)$. Combined with the comparison principle (Corollary 1), we prove the convergence by following Barles and Souganidis (1991).

Lemma 1. The value function $H$ obeys the following bounds:

$$
\begin{gathered}
x+q S-\max _{i} \gamma^{-}(i) \sigma \bar{Q}(T-t)-\Upsilon_{M O} \bar{Q}-\alpha \bar{Q}^{2}-\phi_{q} \bar{Q}^{2}(T-t)-\phi_{f}(T-t) \\
\leq H \leq \\
x+q S+(T-t)\left[\Upsilon_{L O}\left(\lambda^{-} V \max _{i} p_{\text {buy }}(i)+\max _{i} \lambda^{+}(i) p_{\text {sell }}\right)+\max _{i} \gamma^{+}(i) \sigma \bar{Q}\right] .
\end{gathered}
$$

Proof. For a proof see Appendix.

Proposition 1. (Monotonicity) For any $\delta$ t less than some constant, $\varphi_{1}, \varphi_{2} \in C_{b}^{1}([0, T] \times([0, \bar{Q}] \cap$ $\mathbb{Z}) \times\{1,2,3\})$ such that $\varphi_{1} \leq \varphi_{2}$, we have $\mathcal{S}^{\delta t}\left(t, q, Z, \varphi_{1}\right) \leq \mathcal{S}^{\delta t}\left(t, q, Z, \varphi_{2}\right)$.

Proof. From expression 20 we observe that $\mathcal{T}^{\delta t}(t, q, Z, \varphi)$ is monotone non-decreasing in $\varphi$, given

$$
\delta t<\left[(1+\kappa)\left(\lambda^{s} p_{\text {sell }}+\lambda^{-} V \max _{i} p_{\text {buy }}(i)+\max _{i} \lambda^{+}(i) p_{\text {sell }}-G_{Z, Z}\right)\right]^{-1},
$$

and monotonicity of $\mathcal{M}^{\delta t}$ is obvious.

Proposition 2. (Stability) For any $\delta t>0$, there exists a unique solution $h^{\delta t}(t, q, Z)$ to 22 . Furthermore, we have the uniform bounds

$$
L(t, q) \leq h^{\delta t}(t, q, Z) \leq U(t)
$$

where

$$
U(t)=(T-t)\left[\Upsilon_{L O}\left(\lambda^{-} V \max _{i} p_{b u y}(i)+\max _{i} \lambda^{+}(i) p_{\text {sell }}\right)+\max _{i} \gamma^{+}(i) \sigma \bar{Q}\right]
$$


and

$$
L(t, q)=-\max _{i} \gamma^{-}(i) \sigma \bar{Q}(T-t)-\Upsilon_{M O} q-\alpha \bar{Q}^{2}-\phi_{q} \bar{Q}^{2}(T-t)-\phi_{f}(T-t)
$$

Proof. For a proof see Appendix.

Proposition 3. (Consistency) For all $(t, q, Z) \in[0, T) \times([0, \bar{Q}] \cap \mathbb{Z}) \times\{1,2,3\}$ and $\varphi \in C_{b}^{1}([0, T] \times$ $([0, \bar{Q}] \cap \mathbb{Z}) \times\{1,2,3\})$, we have

$$
\begin{aligned}
& \lim _{\substack{\delta t \rightarrow 0 \\
t^{\prime} \rightarrow t}} \frac{1}{\delta t}\left[\mathcal{T}^{\delta t}\left(t^{\prime}+\delta t, q, Z, \varphi\right)-\varphi\left(t^{\prime}, q, Z\right)\right] \\
& =\partial_{t} \varphi-\phi_{q} q^{2} \\
& +\sup _{c \in \mathcal{U}}\left[c \left(-\phi_{f}+\left(\gamma^{+}(1)-\gamma^{-}(1)\right) \sigma q+\lambda^{s} p_{\text {sell }}\left(\Upsilon_{L O}+\varphi(t, q-1, Z)-(1+\kappa) \varphi\right)\right.\right. \\
& \left.+\lambda^{-} p_{\text {buy }}(Z)\left(V \Upsilon_{L O}+\varphi(t, q+V, Z)-(1+\kappa) \varphi\right)\right) \\
& +(1-c)\left(+\left(\gamma^{+}(Z)-\gamma^{-}(Z)\right) \sigma q\right. \\
& \left.\left.+\lambda^{+}(Z) p_{\text {sell }}\left(\Upsilon_{L O}+\varphi(t, q-1, Z)-(1+\kappa) \varphi\right)\right)\right] \\
& +\sum_{K \neq Z}(\varphi(t, q, K)-(1+\kappa) \varphi(t, q, Z)) G_{Z, K},
\end{aligned}
$$

and

$$
\lim _{\substack{\delta t \rightarrow 0 \\ t^{\prime} \rightarrow t}} \mathcal{M}^{\delta t}\left(t^{\prime}+\delta t, q, Z, \varphi\right)=\frac{\varphi(t, q-1, Z)-\Upsilon_{M O}}{1+\kappa}
$$

Proof. The limits converge by directly applying $\varphi \in C_{b}^{1}([0, T] \times([0, \bar{Q}] \cap \mathbb{Z}) \times\{1,2,3\})$.

Theorem 2. (Convergence) The function $h^{\delta t}(t, q, Z)$ converges locally uniformly to the unique viscosity solution $h(t, q, Z)$ as $\delta t \rightarrow 0$.

Proof. Follows Corollary from 1 (see the Appendix), Propositions 1, 2, 3, and Barles and Souganidis (1991). See Appendix for more details. 


\section{Conclusions and Further Research}

In this paper we derived the optimal trading strategy for an investor who employs spoof LOs to improve the rate and prices at which she sells shares with limit orders in an order-driven electronic market. The spoof strategy is illegal and benefits from misleading other market participants about the supply and demand of an asset.

We focused on a strategy where the investor may choose to tilt the volume imbalance of the LOB by posting LOs on one side of the book. The strategy trades off the benefits from spoofing and the potential fine the investor may receive from the financial authorities. When the expected fine is low, we found that spoofing the book considerably increases the financial performance of an execution strategy. The financial improvement stems from: (i) executing more shares using limit orders (instead of market orders that cross the spread and pay fees), (ii) employing sell limit orders to unwind shares that were 'inadvertently' purchased with spoof buy limit orders, and (iii) increasing the drift of the midprice so inventory appreciates and shares are sold at higher prices.

We also showed that spoofing deviates the price of the asset from its fundamental value. This deviation is highest when market participants believe the information conveyed by the LOB and the fine for spoofing is zero. Our simulations showed that at the end of the trading horizon, which lasts five minutes, the mean price of the asset is 52 cents higher than the mean price of the asset in the absence of spoofing. As the penalty for spoofing increases, the investor relies less on spoof LOs, so the manipulation of the price of the asset is less effective and the PnL of the spoofing strategy decreases.

The framework we developed here can be employed to develop other trading strategies that rely on spoof orders to improve their financial performance. For example, a market making strategy that employs sell and buy spoof LOs to open and close positions, respectively. There are other ways to spoof the LOB that could be considered within the framework developed in this paper. We provide three examples:

- Phantom liquidity inside the spread. The investor wishes to sell shares. The strategy consists of sending spoof buy LOs inside the spread (i.e., improve the bid price) to entice other liquidity providers to join the queue at the improved bid price. As soon as other traders send LOs at the new best bid price, the spoofer cancels her spoof buy LOs and sends sell MOs. A similar strategy is used when the investor wishes to purchase shares.

- Cross-spoofing. This is identical to the strategy developed in this paper, only that two (or more) investors agree to spoof the market to avoid being detected by financial authorities. In the strategy, one investor(s) spoofs the LOB and the other investor(s) liquidates the position in the shares.

- Layering. The strategy consists of posting several large LOs at different prices on one side of the book. The goal is to move the price because other market participants interpret the one sided pressure in the LOB as a signal of a price move and trade in anticipation of expected change in price. 
To the best of our knowledge this is the first work that provides a mathematical framework to develop optimal spoofing strategies. Strategies that layer or spoof the book are detrimental to the integrity of markets. Our framework can be employed to understand patterns of spoofing and to develop data techniques (e.g., machine learning) to identify strategies that are detrimental to the market.

\section{References}

\section{References}

Agency for the Cooperation of Energy Regulators (2019a). Layering and spoofin in continuous wholesale energy markets.

Agency for the Cooperation of Energy Regulators (2019b). Remit quarterly.

Alfonsi, A. and J. I. Acevedo (2014). Optimal execution and price manipulations in time-varying limit order books. Applied Mathematical Finance 21(3), 201-237.

Allen, F. and D. Gale (1992). Stock-price manipulation. The Review of Financial Studies 5(3), 503-529.

Barles, G. and P. E. Souganidis (1991). Convergence of approximation schemes for fully nonlinear second order equations. Asymptotic Analysis $\underline{4}(3), 271-283$.

Cao, Y., Y. Li, S. Coleman, A. Belatreche, and T. M. McGinnity (2014). Detecting price manipulation in the financial market. In Computational Intelligence for Financial Engineering \& Economics (CIFEr), 2104 IEEE Conference on, pp. 77-84. IEEE.

Cao, Y., Y. Li, S. A. Coleman, A. Belatreche, T. M. McGinnity, et al. (2015). Adaptive hidden markov model with anomaly states for price manipulation detection. IEEE Trans. Neural Netw. Learning Syst. $26(2), 318-330$.

Cartea, Á., R. Donnelly, and S. Jaimungal (2018). Enhancing trading strategies with order book signals. Applied Mathematical Finance, 1-35.

Cartea, Á. and S. Jaimungal (2015a). Optimal execution with limit and market orders. Quantitative Finance 15(8), 1279-1291.

Cartea, Á. and S. Jaimungal (2015b). Risk metrics and fine tuning of high-frequency trading strategies. Mathematical Finance 25(3), 576-611.

Cartea, Á., S. Jaimungal, and J. Penalva (2015). Algorithmic and high-frequency trading. Cambridge University Press.

Dodd-Frank (2010). Public Law 111-203, reform, Dodd-Frank Wall Street and Act, consumer protection. US Statutes at Large $\underline{124}, 1376$.

Foucault, T. (2012). Pricing liquidity in electronic markets. Foresight Driver Review (DR18) 15(8), $1-26$. 
Guéant, O. (2016). The financial mathematics of market liquidity: From optimal execution to market

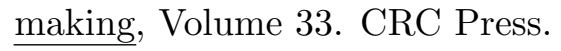

Ishii, H. and S. Koike (1991). Viscosity solutions for monotone systems of second-order elliptic PDEs. Communications in Partial Differential Equations 16(6-7), 1095-1128.

Klöck, F., A. Schied, and Y. Sun (2017). Price manipulation in a market impact model with dark pool. Applied Mathematical Finance 24(5), 417-450.

Lee, E. J., K. S. Eom, and K. S. Park (2013). Microstructure-based manipulation: Strategic behavior and performance of spoofing traders. Journal of Financial Markets 16(2), 227-252.

Øksendal, B. and A. Sulem (2007). Applied Stochastic Control of Jump Diffusions. Springer Science \& Business Media.

Wang, Y. (2015). Strategic spoofing order trading by different types of investors in the futures markets. Wall Street Journal. 


\section{Appendices}

\section{A. Proofs}

\section{A.1. Proof of Theorem 1}

Proof. We follow Øksendal and Sulem (2007). Let the function $f^{c}(q)=-\phi_{q} q^{2}-\phi_{c} c$. We define the operators $\mathfrak{L}^{c}$ and $\mathfrak{M}$ such that the HJBQVI $(12)$ is represented by the form of

$$
\max \left\{\sup _{c \in \mathcal{U}}\left[\mathfrak{L}^{c} \widetilde{h}+f^{c}\right], \mathfrak{M} \widetilde{h}-\widetilde{h}\right\}=0 .
$$

Let $\widetilde{h}$ be the solution to $(12)$ and define the candidate solution $\tilde{H}=x+q S+\widetilde{h}(t, q, Z)$. We want to show that $\tilde{H}=H$.

For any control $(c, \boldsymbol{\tau})$, from Itô's Lemma we have

$$
\begin{aligned}
& \mathbb{E}\left[\tilde{H}\left(\tau_{n}^{-}, X_{\tau_{n}^{-}}^{c, \boldsymbol{\tau}}, S_{\tau_{n}^{-}}^{c}, Q_{\tau_{n}^{-}}^{c, \boldsymbol{\tau}}, Z_{\tau_{n}^{-}}^{c, \boldsymbol{\tau}}\right) \mid \mathcal{F}_{t}\right]-\mathbb{E}\left[\tilde{H}\left(\tau_{n-1}, X_{\tau_{n-1}}^{c, \boldsymbol{\tau}}, S_{\tau_{n-1}}^{c}, Q_{\tau_{n-1}}^{c, \boldsymbol{\tau}}, Z_{\tau_{n-1}}^{c, \boldsymbol{\tau}}\right) \mid \mathcal{F}_{t}\right] \\
& =\mathbb{E}\left[\int_{\tau_{n-1}}^{\tau_{n}} \mathfrak{L} c \hat{h}\left(u, Q_{u}^{c, \boldsymbol{\tau}}, Z_{u}^{c, \boldsymbol{\tau}}\right) \mathrm{d} u \mid \mathcal{F}_{t}\right],
\end{aligned}
$$

and

$$
\begin{aligned}
& \tilde{H}\left(\tau_{n}, X_{\tau_{n}}^{c, \boldsymbol{\tau}}, S_{\tau_{n}}^{c}, Q_{\tau_{n}}^{c, \boldsymbol{\tau}}, Z_{\tau_{n}}^{c, \boldsymbol{\tau}}\right)-\tilde{H}\left(\tau_{n}^{-}, X_{\tau_{n}^{-}}^{c, \boldsymbol{\tau}}, S_{\tau_{n}^{-}}^{c}, Q_{\tau_{n}^{-}}^{c, \boldsymbol{\tau}}, Z_{\tau_{n}^{-}}^{c, \boldsymbol{\tau}}\right) \\
& =\mathfrak{M} \widetilde{h}\left(\tau_{n}^{-}, Q_{\tau_{n}^{-}}^{c, \boldsymbol{\tau}}, Z_{\tau_{n}^{-}}^{c, \boldsymbol{\tau}}\right)-\widetilde{h}\left(\tau_{n}^{-}, Q_{\tau_{n}^{-}}^{c, \boldsymbol{\tau}}, Z_{\tau_{n}^{-}}^{c, \boldsymbol{\tau}}\right)
\end{aligned}
$$

where, with a slight abuse of notation, we only include the jumps from the control. Summing over $\left[t, \tau_{\mathcal{S}} \wedge T\right]$, taking an expectation conditional on $\mathcal{F}_{t}$ and rearranging yields

$$
\begin{aligned}
\tilde{H}(t, x, S, q, Z)= & \mathbb{E}\left[\tilde{H}\left(\tau_{\mathcal{S}} \wedge T, X_{\tau_{\mathcal{S}} \wedge T}^{c, \boldsymbol{\tau}}, S_{\tau_{\mathcal{S}} \wedge T}^{c}, Q_{\tau_{\mathcal{S}} \wedge T}^{c, \boldsymbol{\tau}}, Z_{\tau_{\mathcal{S}} \wedge T}^{c, \boldsymbol{\tau}}\right) \mid \mathcal{F}_{t}\right] \\
& -\mathbb{E}\left[\int_{t}^{\tau_{\mathcal{S}} \wedge T} \mathfrak{L}^{c} \widetilde{h}\left(u, Q_{u}^{c, \boldsymbol{\tau}}, Z_{u}^{c, \boldsymbol{\tau}}\right) \mathrm{d} u \mid \mathcal{F}_{t}\right] \\
& -\mathbb{E}\left[\sum_{\tau_{n} \leq \tau_{\mathcal{S}} \wedge T} \mathfrak{M} \tilde{h}\left(\tau_{n}^{-}, Q_{\tau_{n}^{-}}^{c, \boldsymbol{\tau}}, Z_{\tau_{n}^{-}}^{c, \boldsymbol{\tau}}\right)-\widetilde{h}\left(\tau_{n}^{-}, Q_{\tau_{n}^{-}}^{c, \boldsymbol{\tau}}, Z_{\tau_{n}^{-}}^{c, \boldsymbol{\tau}}\right) \mid \mathcal{F}_{t}\right]
\end{aligned}
$$


From HJBQVI (12) we have

$$
\begin{aligned}
\tilde{H}(t, x, S, q, Z) \geq \mathbb{E}\left[\tilde{H}\left(\tau_{\mathcal{S}} \wedge T, X_{\tau_{\mathcal{S}} \wedge T}^{c, \boldsymbol{\tau}}, S_{\tau_{\mathcal{S}} \wedge T}^{c}, Q_{\tau_{\mathcal{S}} \wedge T}^{c, \boldsymbol{\tau}}, Z_{\tau_{\mathcal{S}} \wedge T}^{c, \boldsymbol{\tau}}\right)\right. & \\
& \left.-\phi_{q} \int_{t}^{\tau_{\mathcal{S}} \wedge T}\left(Q_{u}^{c, \boldsymbol{\tau}}\right)^{2} \mathrm{~d} u-\phi_{f} \int_{t}^{\tau_{\mathcal{S}} \wedge T} c_{u} \mathrm{~d} u \mid \mathcal{F}_{t}\right] \\
=\mathbb{E}\left[X_{\tau_{\mathcal{S}} \wedge T}^{c, \boldsymbol{\tau}}\right. & +Q_{\tau_{\mathcal{S}} \wedge T}^{c, \tau}\left(S_{\tau_{\mathcal{S}} \wedge T}^{c}-\Upsilon_{M O}-\alpha Q_{\tau_{\mathcal{S}} \wedge T}^{c, \boldsymbol{\tau}}\right) \\
& \left.-\phi_{q} \int_{t}^{\tau_{\mathcal{S}} \wedge T}\left(Q_{u}^{c, \boldsymbol{\tau}}\right)^{2} \mathrm{~d} u-\phi_{f} \int_{t}^{\tau_{\mathcal{S}} \wedge T} c_{u} \mathrm{~d} u \mid \mathcal{F}_{t}\right] .
\end{aligned}
$$

Since the inequality above holds for any control $(c, \boldsymbol{\tau})$, we have

$$
\begin{aligned}
& \tilde{H}(t, x, S, q, Z) \geq \sup _{\left(c_{t}, \boldsymbol{\tau}\right) \in \mathcal{A}} \mathbb{E}_{t, x, S, q, Z}\left[X_{\tau_{\mathcal{S}} \wedge T}^{c, \boldsymbol{\tau}}+Q_{\tau_{\mathcal{S}} \wedge T}^{c, \boldsymbol{\tau}}\left(S_{\tau_{\mathcal{S}} \wedge T}^{c}-\Upsilon_{M O}-\alpha Q_{\tau_{\mathcal{S}} \wedge T}^{c, \boldsymbol{\tau}}\right)\right. \\
& \left.-\phi_{q} \int_{t}^{\tau_{\mathcal{S}} \wedge T}\left(Q_{u}^{c, \boldsymbol{\tau}}\right)^{2} \mathrm{~d} u-\phi_{f} \int_{t}^{\tau^{\mathcal{S}} \wedge T} c_{u} \mathrm{~d} u\right] \\
& =H(t, x, S, q, Z) \text {. }
\end{aligned}
$$

Now if we use the optimal control $\left(c^{*}, \tau^{*}\right), 27$ becomes

$$
\begin{aligned}
\tilde{H}(t, x, S, q, Z)= & \mathbb{E}\left[\tilde{H}\left(\tau_{\mathcal{S}} \wedge T, X_{\tau_{\mathcal{S}} \wedge T}^{c^{*}, \boldsymbol{\tau}^{*}}, S_{\tau_{\mathcal{S}} \wedge T}^{c^{*}}, Q_{\tau_{\mathcal{S}} \wedge T}^{c^{*}, \boldsymbol{\tau}^{*}}, Z_{\tau_{\mathcal{S}} \wedge T}^{c^{*}, \boldsymbol{\tau}^{*}}\right)\right. \\
& \left.\quad-\phi_{q} \int_{t}^{\tau_{\mathcal{S}} \wedge T}\left(Q_{u}^{c^{*}, \tau^{*}}\right)^{2} \mathrm{~d} u-\phi_{f} \int_{t}^{\tau_{\mathcal{S}} \wedge T} c_{u}^{*} \mathrm{~d} u \mid \mathcal{F}_{t}\right] \\
= & \mathbb{E}\left[X_{\tau_{\mathcal{S}} \wedge T}^{c^{*}, \boldsymbol{\tau}^{*}}+Q_{\tau_{\mathcal{S}} \wedge T}^{c^{*}, \tau^{*}}\left(S_{\tau_{\mathcal{S}} \wedge T}^{c^{*}}-\Upsilon_{M O}-\alpha Q_{\tau_{\mathcal{S}} \wedge T}^{c^{*}, \tau^{*}}\right)\right. \\
& \left.-\phi_{q} \int_{t}^{\tau_{\mathcal{S}} \wedge T}\left(Q_{u}^{c^{*}, \tau^{*}}\right)^{2} \mathrm{~d} u-\phi_{f} \int_{t}^{\tau_{\mathcal{S}} \wedge T} c_{u}^{*} \mathrm{~d} u \mid \mathcal{F}_{t}\right] \\
\leq & \sup _{\left(c_{t}, \boldsymbol{\tau}\right) \in \mathcal{A}} \mathbb{E}_{t, x, S, q, Z}\left[X_{\tau_{\mathcal{S}} \wedge T}^{c, \boldsymbol{\tau}}+Q_{\tau_{\mathcal{S}} \wedge T}^{c, \boldsymbol{\tau}}\left(S_{\tau_{\mathcal{S}} \wedge T}^{c}-\Upsilon_{M O}-\alpha Q_{\tau_{\mathcal{S}} \wedge T}^{c, \boldsymbol{\tau}}\right)\right. \\
& \left.-\phi_{q} \int_{t}^{\tau_{\mathcal{S}} \wedge T}\left(Q_{u}^{c, \boldsymbol{\tau}}\right)^{2} \mathrm{~d} u-\phi_{f} \int_{t}^{\tau_{\mathcal{S}} \wedge T} c_{u} \mathrm{~d} u\right] \\
= & H(t, x, S, q, Z) .
\end{aligned}
$$

Hence $H=\hat{H}$. 


\section{A.2. Comparison principle}

In this section we prove the comparison principle of $[12$, which we employ later in the proof of convergence of the numerical scheme. We introduce a regularised version of (12) and prove the comparison principle of this regularised version. The proof of convergence of the numerical scheme of the unregularised version is beyond the scope of this work.

The regularised version of HJBQVI 12 is

$$
\begin{array}{r}
\max \left\{\partial_{t} h-\phi_{q} q^{2}\right. \\
+\sup _{c \in \mathcal{U}}\left[c \left(-\phi_{f}+\left(\gamma^{+}(1)-\gamma^{-}(1)\right) \sigma q+\lambda^{s} p_{\text {sell }}\left(\Upsilon_{L O}+h(t, q-1, Z)-(1+\kappa) h\right)\right.\right. \\
\left.+\lambda^{-} p_{\text {buy }}(Z)\left(V \Upsilon_{L O}+h(t, q+V, Z)-(1+\kappa) h\right)\right) \\
+(1-c)\left(\left(\gamma^{+}(Z)-\gamma^{-}(Z)\right) \sigma q\right. \\
\left.\left.+\lambda^{+}(Z) p_{\text {sell }}\left(\Upsilon_{L O}+h(t, q-1, Z)-(1+\kappa) h\right)\right)\right] \\
+\sum_{K \neq Z}(h(t, q, K)-(1+\kappa) h(t, q, Z)) G_{Z, K}, \\
\left.-\Upsilon_{M O}+h(t, q-1, Z)-(1+\kappa) h\right\}=0,
\end{array}
$$

where $\kappa \downarrow 0$ is a robustness parameter, with terminal and boundary conditions

$$
h(T, q, Z)=q\left(-\Upsilon_{M O}-\alpha q\right) \quad h(t, 0, Z)=0
$$

respectively.

For notational convenience we make the following changes of variables:

$$
s=T-t, \quad g_{q, Z}(s)=-h(t, q, Z), \quad d_{q, Z}(s)=c(t, q, Z),
$$

and define $\mathcal{U}_{d}$ to be the set of admissible strategies in the new variables, and let

$$
F_{q, Z}(r, u, p)=\max \left\{P_{q, Z}(r, u, p), O_{q, Z}(r, u, p)\right\}
$$


where

$$
\begin{aligned}
& P_{q, Z}(r, u, p)=p-\phi_{q} q^{2} \\
& +\sup _{d_{q, Z}(r) \in \mathcal{U}_{d}}\left[d _ { q , Z } ( r ) \left(-\phi_{f}+\left(\gamma^{+}(1)-\gamma^{-}(1)\right) \sigma q\right.\right. \\
& +\lambda^{s} p_{\text {sell }}\left(\Upsilon_{L O}-u_{q-1, Z}+(1+\kappa) u_{q, Z}\right) \\
& \left.+\lambda^{-} p_{b u y}(Z)\left(V \Upsilon_{L O}-u_{q+V, Z}+(1+\kappa) u_{q, Z}\right)\right) \\
& +\left(1-d_{q, Z}(r)\right) \lambda^{+}(Z)\left(\left(\gamma^{+}(Z)-\gamma^{-}(Z)\right) \sigma q\right. \\
& \left.\left.+p_{\text {sell }}\left(\Upsilon_{L O}-u_{q-1, Z}+(1+\kappa) u_{q, Z}\right)\right)\right] \\
& -\sum_{K \neq Z}\left(u_{q, K}-(1+\kappa) u_{q, Z}\right) G_{Z, K},
\end{aligned}
$$

and

$$
O_{q, Z}(r, u, p)=(1+\kappa) u_{q, Z}-\left(\Upsilon_{M O}+u_{q-1, Z}\right)
$$

Then, the HJBQVI becomes

$$
F_{q, Z}\left(s, g, \partial_{s} g\right)=0
$$

with terminal and boundary conditions

$$
g_{q, Z}(0)=q\left(\Upsilon_{M O}+\alpha q\right), \quad g_{0, Z}(t)=0,
$$

respectively.

Proposition 4. (A1) Let $u=\left(u_{q, Z}\right)$ and $v=\left(v_{q, Z}\right)$. Suppose that $u_{q^{*}, Z^{*}}-v_{q^{*}, Z^{*}}=\max _{q, Z}\left\{u_{q, Z}-\right.$ $\left.v_{q, Z}\right\}$, then there exists $c_{0}>0$, where

$$
c_{0}=\kappa \min \left\{\lambda^{s} p_{\text {sell }}+\lambda^{-} p_{\text {buy }}\left(Z^{*}\right), \lambda^{+}\left(Z^{*}\right) p_{\text {sell }},-G_{Z^{*}, Z^{*}}, 1\right\},
$$

such that

$$
F_{q^{*}, Z^{*}}(s, u, p)-F_{q^{*}, Z^{*}}(s, v, p) \geq c_{0}\left(u_{q^{*}, Z^{*}}-v_{q^{*}, Z^{*}}\right) .
$$

(A2) There exists $\beta>1$ and a continuous function $\omega: \mathbb{R}_{+} \rightarrow \mathbb{R}_{+}, \omega(0)=0$, such that

$$
F_{q, Z}\left(s, u, \beta\left(s^{\prime}-s\right)\right)-F_{q, Z}\left(s^{\prime}, u, \beta\left(s^{\prime}-s\right)\right) \leq \omega\left(\beta\left(s^{\prime}-s\right)^{2}+\beta^{-1}\right)
$$

for all $q, Z$. 
Proof. (A1) The first required inequality is stated as follows:

$\max \left\{P_{q^{*}, Z^{*}}(s, u, p), O_{q^{*}, Z^{*}}(s, u, p)\right\}-\max \left\{P_{q^{*}, Z^{*}}(s, v, p), O_{q^{*}, Z^{*}}(s, v, p)\right\} \geq c_{0}\left(u_{q^{*}, Z^{*}}-v_{q^{*}, Z^{*}}\right)$.

We only need to prove the following two inequalities:

$$
\begin{aligned}
& P_{q^{*}, Z^{*}}(s, u, p)-P_{q^{*}, Z^{*}}(s, v, p) \geq c_{0}\left(u_{q^{*}, Z^{*}}-v_{q^{*}, Z^{*}}\right), \\
& O_{q^{*}, Z^{*}}(s, u, p)-O_{q^{*}, Z^{*}}(s, v, p) \geq c_{0}\left(u_{q^{*}, Z^{*}}-v_{q^{*}, Z^{*}}\right) .
\end{aligned}
$$

The other two situations are converted to the two inequalities above. If we obtain different terms from the maximum, then we have

$$
\begin{aligned}
& O_{q^{*}, Z^{*}}(s, u, p)-P_{q^{*}, Z^{*}}(s, v, p) \geq P_{q^{*}, Z^{*}}(s, u, p)-P_{q^{*}, Z^{*}}(s, v, p), \\
& P_{q^{*}, Z^{*}}(s, u, p)-O_{q^{*}, Z^{*}}(s, v, p) \geq O_{q^{*}, Z^{*}}(s, u, p)-O_{q^{*}, Z^{*}}(s, v, p) .
\end{aligned}
$$

For the first inequality,

$$
\begin{aligned}
& P_{q^{*}, Z^{*}}(s, u, p)-P_{q^{*}, Z^{*}}(s, v, p) \\
& =\max \left[-\phi_{f}+\left(\gamma^{+}(1)-\gamma^{-}(1)\right) \sigma q+\lambda^{s} p_{\text {sell }}\left(\Upsilon_{L O}-u_{q^{*}-1, Z^{*}}+(1+\kappa) u_{q^{*}, Z^{*}}\right)\right. \\
& +\lambda^{-} p_{\text {buy }}\left(Z^{*}\right)\left(V \Upsilon_{L O}-u_{q^{*}+V, Z^{*}}+(1+\kappa) u_{q^{*}, Z^{*}}\right), \\
& \left.\left(\gamma^{+}(Z)-\gamma^{-}(Z)\right) \sigma q+\lambda^{+}\left(Z^{*}\right) p_{\text {sell }}\left(\Upsilon_{L O}-u_{q^{*}-1, Z^{*}}+(1+\kappa) u_{q^{*}, Z^{*}}\right)\right] \\
& -\max \left[-\phi_{f}+\left(\gamma^{+}(1)-\gamma^{-}(1)\right) \sigma q+\lambda^{s} p_{\text {sell }}\left(\Upsilon_{L O}-v_{q^{*}-1, Z^{*}}+(1+\kappa) v_{q^{*}, Z^{*}}\right)\right. \\
& +\lambda^{-} p_{\text {buy }}\left(Z^{*}\right)\left(V \Upsilon_{L O}-v_{q^{*}+V, Z^{*}}+(1+\kappa) v_{q^{*}, Z^{*}}\right), \\
& \left.\left(\gamma^{+}(Z)-\gamma^{-}(Z)\right) \sigma q+\lambda^{+}\left(Z^{*}\right) p_{\text {sell }}\left(\Upsilon_{L O}-v_{q^{*}-1, Z^{*}}+(1+\kappa) v_{q^{*}, Z^{*}}\right)\right] \\
& +\sum_{K \neq Z^{*}}\left((1+\kappa)\left(u_{q^{*}, Z^{*}}-v_{q^{*}, Z^{*}}\right)-\left(u_{q^{*}, K}-v_{q^{*}, K}\right)\right) G_{Z^{*}, K}
\end{aligned}
$$

For the first two maxima we use the same technique as above and only need to prove that

$$
\begin{aligned}
& \lambda^{s} p_{\text {sell }}\left(\Upsilon_{L O}-u_{q^{*}-1, Z^{*}}+(1+\kappa) u_{q^{*}, Z^{*}}\right)+\lambda^{-} p_{\text {buy }}\left(Z^{*}\right)\left(V \Upsilon_{L O}-u_{q^{*}+V, Z^{*}}+(1+\kappa) u_{q^{*}, Z^{*}}\right) \\
& \quad-\lambda^{s} p_{\text {sell }}\left(\Upsilon_{L O}-v_{q^{*}-1, Z^{*}}+(1+\kappa) v_{q^{*}, Z^{*}}\right)-\lambda^{-} p_{b u y}\left(Z^{*}\right)\left(V \Upsilon_{L O}-v_{q^{*}+V, Z^{*}}+(1+\kappa) v_{q^{*}, Z^{*}}\right) \\
& \geq c_{0}\left(u_{q^{*}, Z^{*}}-v_{q^{*}, Z^{*}}\right),
\end{aligned}
$$


and

$$
\begin{aligned}
& \lambda^{+}\left(Z^{*}\right) p_{\text {sell }}\left(\Upsilon_{L O}-u_{q^{*}-1, Z^{*}}+(1+\kappa) u_{q^{*}, Z^{*}}\right)-\lambda^{+}\left(Z^{*}\right) p_{\text {sell }}\left(\Upsilon_{L O}-v_{q^{*}-1, Z^{*}}+(1+\kappa) v_{q^{*}, Z^{*}}\right) \\
& \quad \geq c_{0}\left(u_{q^{*}, Z^{*}}-v_{q^{*}, Z^{*}}\right) .
\end{aligned}
$$

To prove inequality (37), we write

$$
\begin{aligned}
& \lambda^{s} p_{\text {sell }}\left(\Upsilon_{L O}-u_{q^{*}-1, Z^{*}}+(1+\kappa) u_{q^{*}, Z^{*}}\right)+\lambda^{-} p_{\text {buy }}\left(Z^{*}\right)\left(V \Upsilon_{L O}-u_{q^{*}+V, Z^{*}}+(1+\kappa) u_{q^{*}, Z^{*}}\right) \\
& \quad-\lambda^{s} p_{\text {sell }}\left(\Upsilon_{L O}-v_{q^{*}-1, Z^{*}}+(1+\kappa) v_{q^{*}, Z^{*}}\right)-\lambda^{-} p_{\text {buy }}\left(Z^{*}\right)\left(V \Upsilon_{L O}-v_{q^{*}+V, Z^{*}}+(1+\kappa) v_{q^{*}, Z^{*}}\right) \\
& =\lambda^{s} p_{\text {sell }}\left((1+\kappa)\left(u_{q^{*}, Z^{*}}-v_{q^{*}, Z^{*}}\right)-\left(u_{q^{*}-1, Z^{*}}-v_{q^{*}-1, Z^{*}}\right)\right) \\
& \quad+\lambda^{-} p_{\text {buy }}\left(Z^{*}\right)\left((1+\kappa)\left(u_{q^{*}, Z^{*}}-u_{q^{*}, Z^{*}}\right)-\left(u_{q^{*}+V, Z^{*}}-v_{q^{*}+V, Z^{*}}\right)\right) \\
& \geq \kappa\left(\lambda^{s} p_{\text {sell }}+\lambda^{-} p_{\text {buy }}\left(Z^{*}\right)\right)\left(u_{q^{*}, Z^{*}}-u_{q^{*}, Z^{*}}\right) \\
& \geq c_{0}\left(u_{q^{*}, Z^{*}}-v_{q^{*}, Z^{*}}\right),
\end{aligned}
$$

by the definition of $\left(q^{*}, Z^{*}\right)$ in $(\mathrm{A} 1)$.

To prove inequality (38), we write

$$
\begin{aligned}
& \lambda^{+}\left(Z^{*}\right) p_{\text {sell }}\left(\Upsilon_{L O}-u_{q^{*}-1, Z^{*}}+(1+\kappa) u_{q^{*}, Z^{*}}\right)-\lambda^{+}\left(Z^{*}\right) p_{\text {sell }}\left(\Upsilon_{L O}-v_{q^{*}-1, Z^{*}}+(1+\kappa) v_{q^{*}, Z^{*}}\right) \\
& =\lambda^{+}\left(Z^{*}\right) p_{\text {sell }}\left((1+\kappa)\left(u_{q^{*}, Z^{*}}-v_{q^{*}, Z^{*}}\right)-\left(u_{q^{*}-1, Z^{*}}-v_{q^{*}-1, Z^{*}}\right)\right) \\
& \geq \kappa \lambda^{+}\left(Z^{*}\right) p_{\text {sell }}\left(u_{q^{*}, Z^{*}}-v_{q^{*}, Z^{*}}\right) \\
& \geq c_{0}\left(u_{q^{*}, Z^{*}}-v_{q^{*}, Z^{*}}\right)
\end{aligned}
$$

by the definition of $\left(q^{*}, Z^{*}\right)$ in $(\mathrm{A} 1)$.

We also have

$$
\begin{aligned}
\sum_{K \neq Z^{*}}\left((1+\kappa)\left(u_{q^{*}, Z^{*}}-v_{q^{*}, Z^{*}}\right)-\left(u_{q^{*}, K}-v_{q^{*}, K}\right)\right) G_{Z^{*}, K} & \geq \kappa \sum_{K \neq Z^{*}}\left(u_{q^{*}, Z^{*}}-v_{q^{*}, Z^{*}}\right) G_{Z^{*}, K} \\
& =\kappa\left(-G_{Z^{*}, Z^{*}}\right)\left(u_{q^{*}, Z^{*}}-v_{q^{*}, Z^{*}}\right) \\
& \geq c_{0}\left(u_{q^{*}, Z^{*}}-v_{q^{*}, Z^{*}}\right)
\end{aligned}
$$

by the definition of $\left(q^{*}, Z^{*}\right)$ in (A1). Thus $P_{q^{*}, Z^{*}}(s, u, p)-P_{q^{*}, Z^{*}}(s, v, p) \geq c_{0}\left(u_{q^{*}, Z^{*}}-v_{q^{*}, Z^{*}}\right)$. 


$$
\begin{aligned}
& O_{q^{*}, Z^{*}}(s, u, p)-O_{q^{*}, Z^{*}}(s, v, p) \\
& \quad=\left((1+\kappa) u_{q^{*}, Z^{*}}-\left(\Upsilon_{M O}+u_{q^{*}-1, Z^{*}}\right)\right)-\left((1+\kappa) v_{q^{*}, Z^{*}}-\left(\Upsilon_{M O}+v_{q^{*}-1, Z^{*}}\right)\right) \\
& \quad=(1+\kappa)\left(u_{q^{*}, Z^{*}}-v_{q^{*}, Z^{*}}\right)-\left(u_{q^{*}-1, Z^{*}}-v_{q^{*}-1, Z^{*}}\right) \\
& \quad \geq \kappa\left(u_{q^{*}, Z^{*}}-v_{q^{*}, Z^{*}}\right) \\
& \quad \geq c_{0}\left(u_{q^{*}, Z^{*}}-v_{q^{*}, Z^{*}}\right),
\end{aligned}
$$

by the definition of $\left(q^{*}, Z^{*}\right)$ in $(\mathrm{A} 1)$. Hence, we obtain

$$
F_{q^{*}, Z^{*}}(s, u, p)-F_{q^{*}, Z^{*}}(s, v, p) \geq c_{0}\left(u_{q^{*}, Z^{*}}-v_{q^{*}, Z^{*}}\right) .
$$

(A2) Inequality (36) is satisfied because

$$
F_{q, Z}\left(s, u, \beta\left(s^{\prime}-s\right)\right)-F_{q, Z}\left(s^{\prime}, u, \beta\left(s^{\prime}-s\right)\right)=0 .
$$

Theorem 3. (Comparison Principle) Assume $F$ is continuous. Let $g_{1}$ and $g_{2}$ be, respectively, a bounded subsolution and a bounded supersolution of (33). Suppose that $g_{1}(0) \leq g_{2}(0)$. Then $g_{1} \leq g_{2}$.

Proof. See Theorem 4.7 in Ishii and Koike (1991).

Corollary 1. Equation (31) admits a comparison principle.

Proof. Directly from Theorem 3 by change of variables. 


\section{A.3. Proof of Lemma 1}

Proof.

$$
\begin{aligned}
& H(t, x, S, q, Z) \\
& =\sup _{\left(c_{t}, \boldsymbol{\tau}\right) \in \mathcal{A}} \mathbb{E}_{t, x, S, q, Z}\left[X_{\tau_{\mathcal{S}} \wedge T}^{c, \boldsymbol{\tau}}+Q_{\tau_{\mathcal{S}} \wedge T}^{c, \boldsymbol{\tau}}\left(S_{\tau_{\mathcal{S}} \wedge T}^{c}-\Upsilon_{M O}-\alpha Q_{\tau_{\mathcal{S}} \wedge T}^{c, \boldsymbol{\tau}}\right)\right. \\
& \left.-\phi_{q} \int_{t}^{\tau_{\mathcal{S}} \wedge T}\left(Q_{u}^{c, \boldsymbol{\tau}}\right)^{2} \mathrm{~d} u-\phi_{f} \int_{t}^{\tau_{\mathcal{S}} \wedge T} c_{u} \mathrm{~d} u\right] \\
& =x+q\left(S-\Upsilon_{M O}\right) \\
& +\sup _{\left(c_{t}, \boldsymbol{\tau}\right) \in \mathcal{A}} \mathbb{E}_{t, x, S, q, Z}\left[\int_{t}^{\tau_{\mathcal{S}} \wedge T} \mathrm{~d} X_{u}^{c, \boldsymbol{\tau}}+\int_{t}^{\tau_{\mathcal{S}} \wedge T} S_{u}^{c} \mathrm{~d} Q_{u}^{c, \boldsymbol{\tau}}-\Upsilon_{M O}\left(Q_{\tau_{\mathcal{S}} \wedge T}^{c, \boldsymbol{\tau}}-q\right)+\int_{t}^{\tau_{\mathcal{S}} \wedge T} Q_{u}^{c, \boldsymbol{\tau}} \mathrm{d} S_{u}^{c}\right. \\
& \left.-\alpha\left(Q_{\tau_{\mathcal{S}} \wedge T}^{c, \tau}\right)^{2}-\phi_{q} \int_{t}^{\tau_{\mathcal{S}} \wedge T}\left(Q_{u}^{c, \tau}\right)^{2} \mathrm{~d} u-\phi_{f} \int_{t}^{\tau_{\mathcal{S}} \wedge T} c_{u} \mathrm{~d} u\right] \\
& =x+q\left(S-\Upsilon_{M O}\right) \\
& +\sup _{\left(c_{t}, \boldsymbol{\tau}\right) \in \mathcal{A}} \mathbb{E}_{t, x, S, q, Z}\left[\int_{t}^{\tau_{\mathcal{S}} \wedge T}\left(S_{u}^{c}+\Upsilon_{L O}\right) \mathrm{d} N_{u}^{+, c}-\int_{t}^{\tau_{\mathcal{S}} \wedge T}\left(S_{u}^{c}-\Upsilon_{L O}\right) \mathrm{d} N_{u}^{-, c}+\int_{t}^{\tau_{\mathcal{S}} \wedge T}\left(S_{u}^{c}-\Upsilon_{M O}\right) \mathrm{d} M_{u}\right. \\
& +\int_{t}^{\tau_{\mathcal{S}} \wedge T} S_{u}^{c} \mathrm{~d} N_{u}^{-, c}-\int_{t}^{\tau_{\mathcal{S}} \wedge T} S_{u}^{c} \mathrm{~d} N_{u}^{+, c}-\int_{t}^{\tau_{\mathcal{S}} \wedge T} S_{u}^{c} \mathrm{~d} M_{u}-\Upsilon_{M O}\left(Q_{\tau_{\mathcal{S}} \wedge T}^{c, \boldsymbol{\tau}}-q\right) \\
& \left.+\int_{t}^{\tau_{\mathcal{S}} \wedge T} Q_{u}^{c, \boldsymbol{\tau}} \mathrm{d} S_{u}-\alpha\left(Q_{\tau_{\mathcal{S}} \wedge T}^{c, \boldsymbol{\tau}}\right)^{2}-\phi_{q} \int_{t}^{\tau_{\mathcal{S}} \wedge T}\left(Q_{u}^{c, \boldsymbol{\tau}}\right)^{2} \mathrm{~d} u-\phi_{f} \int_{t}^{\tau_{\mathcal{S}} \wedge T} c_{u} \mathrm{~d} u\right] .
\end{aligned}
$$

After cancelling terms, we have

$$
\begin{aligned}
& H(t, x, S, q, Z) \\
& =x+q S \\
& +\sup _{\left(c_{t}, \boldsymbol{\tau}\right) \in \mathcal{A}} \mathbb{E}_{t, x, S, q, Z}\left[\Upsilon_{L O}\left(\int_{t}^{\tau_{\mathcal{S}} \wedge T} \mathrm{~d} N_{u}^{+, c}+\int_{t}^{\tau_{\mathcal{S}} \wedge T} \mathrm{~d} N_{u}^{-, c}\right)+\int_{t}^{\tau_{\mathcal{S}} \wedge T} Q_{u}^{c, \boldsymbol{\tau}} \mathrm{d} S_{u}^{c}\right. \\
& \left.\quad-\Upsilon_{M O}\left(Q_{\tau_{\mathcal{S}} \wedge T}^{c, \boldsymbol{\tau}}+M_{\tau_{\mathcal{S}} \wedge T}-M_{t}\right)-\alpha\left(Q_{\tau_{\mathcal{S}} \wedge T}^{c, \boldsymbol{\tau}}\right)^{2}-\phi_{q} \int_{t}^{\tau_{\mathcal{S}} \wedge T}\left(Q_{u}^{c, \boldsymbol{\tau}}\right)^{2} \mathrm{~d} u-\phi_{f} \int_{t}^{\tau_{\mathcal{S}} \wedge T} c_{u} \mathrm{~d} u\right] .
\end{aligned}
$$

So far we have not introduced any enlargement in the value function, and we give a brief interpretation of each term. The first two integrals, which are non-negative, represent the profit of posting the LOs and earning the spread. The terms

$$
-\Upsilon_{M O}\left(Q_{\tau_{\mathcal{S}} \wedge T}^{c, \boldsymbol{\tau}}+M_{\tau_{\mathcal{S}} \wedge T}-M_{t}\right)-\alpha\left(Q_{\tau_{\mathcal{S}} \wedge T}^{c, \boldsymbol{\tau}}\right)^{2}-\phi_{q} \int_{t}^{\tau_{\mathcal{S}} \wedge T}\left(Q_{u}^{c, \boldsymbol{\tau}}\right)^{2} \mathrm{~d} u-\phi_{f} \int_{t}^{\tau_{\mathcal{S}} \wedge T} c_{u} \mathrm{~d} u
$$

represent the cost of sending MOs, the terminal and running penalties on inventory of the value function, and the fine of spoofing respectively. 
For the lower bound, we first restrict the set of admissible strategies such that the investor does not submit any MOs, which is clearly admissible and hence has performance criteria less than $H$. Also, we drop the three non-negative integrals, and since $c, Q$ and $\tau_{\mathcal{S}}$ are bounded, we have

$$
\begin{aligned}
& H(t, x, S, q, Z) \\
& \geq x+q S \\
& \quad+\sup _{\left(c_{t}, \boldsymbol{\tau}\right) \in \mathcal{A}} \mathbb{E}_{t, x, S, q, Z}\left[\Upsilon_{L O}\left(\int_{t}^{\tau_{\mathcal{S}} \wedge T} \mathrm{~d} N_{u}^{+, c}+\int_{t}^{\tau_{\mathcal{S}} \wedge T} \mathrm{~d} N_{u}^{-, c}\right)+\int_{t}^{\tau_{\mathcal{S}} \wedge T} Q_{u}^{c, \boldsymbol{\tau}} \mathrm{d} S_{u}^{c}\right. \\
& \left.\quad-\Upsilon_{M O} Q_{\tau_{\mathcal{S}}^{c} \wedge T}^{c, \boldsymbol{\tau}}-\alpha\left(Q_{\tau_{\mathcal{S}} \wedge T}^{c, \boldsymbol{\tau}}\right)^{2}-\phi_{q} \int_{t}^{\tau_{\mathcal{S}} \wedge T}\left(Q_{u}^{c, \boldsymbol{\tau}}\right)^{2} \mathrm{~d} u-\phi_{f} \int_{t}^{\tau_{\mathcal{S}} \wedge T} c_{u} \mathrm{~d} u\right] \\
& \geq x+q S-\max _{i} \gamma^{-}(i) \sigma \bar{Q}(T-t)-\Upsilon_{M O} \bar{Q}-\alpha \bar{Q}^{2}-\phi_{q} \bar{Q}^{2}(T-t)-\phi_{f}(T-t) .
\end{aligned}
$$

For the upper bound, we first drop all the non-positive terms, and by enlarging $\tau_{\mathcal{S}} \wedge T$ and $c_{u}$, we obtain

$$
\begin{aligned}
& H(t, x, S, q, Z) \\
& \leq x+q S \\
& \quad+\sup _{\left(c_{t}, \boldsymbol{\tau}\right) \in \mathcal{A}} \mathbb{E}_{t, x, S, q, Z}\left[\Upsilon_{L O}\left(\int_{t}^{\tau_{\mathcal{S}} \wedge T} \mathrm{~d} N_{u}^{+, c}+\int_{t}^{\tau_{\mathcal{S}} \wedge T} \mathrm{~d} N_{u}^{-,, c}\right)+\int_{t}^{\tau_{\mathcal{S}} \wedge T} Q_{u}^{c, \boldsymbol{\tau}} \mathrm{d} S_{u}^{c}\right] \\
& \leq x+q S+(T-t)\left[\Upsilon_{L O}\left(\lambda^{-} V \max _{i} p_{b u y}(i)+\max _{i} \lambda^{+}(i) p_{\text {sell }}\right)+\max _{i} \gamma^{+}(i) \sigma \bar{Q}\right] .
\end{aligned}
$$




\section{A.4. Proof of Proposition 2}

Proof. Existence and uniqueness follow immediately from the definition of the explicit scheme (22). We first prove the upper bound. We notice that $h^{\delta t}(T, q, Z)=-q\left(\Upsilon_{M O}+\alpha q\right) \leq U(T)=0$, and

$$
\begin{array}{r}
\mathcal{T}^{\delta t}(t, q, Z, U)=U(t)+\delta t\left[\begin{array}{l}
- \\
\quad \phi_{q} q^{2}
\end{array}\right. \\
+\sup _{c \in \mathcal{U}}\left[c \left(-\phi_{f}+\left(\gamma^{+}(1)-\gamma^{-}(1)\right) \sigma q+\lambda^{s} p_{\text {sell }}\left(\Upsilon_{L O}-\kappa U(t)\right)\right.\right. \\
+(1-c)\left(\left(\gamma^{+}(Z)-\gamma^{-}(Z)\right) \sigma q\right. \\
\left.+\lambda^{+}(Z) p_{\text {sell }}(Z)\left(V \Upsilon_{L O}-\kappa U(t)\right)\right)
\end{array}
$$

Together with

$$
\mathcal{M}^{\delta t}(t, q, Z, U)=\frac{U(t)-\Upsilon_{M O}}{1+\kappa} \leq U(t) \leq U(t-\delta t)
$$

we have

$$
\mathcal{S}^{\delta t}(t, q, Z, U) \leq U(t-\delta t)
$$

As $h^{\delta t}(t-\delta t, q, Z)=\mathcal{S}^{\delta t}\left(t, q, Z, h^{\delta t}(t, q, Z)\right)$, we prove by induction that $h^{\delta t}(t, q, Z) \leq U(t)$.

Similarly for the lower bound, we have $h^{\delta t}(T, q, Z)=-q\left(\Upsilon_{M O}+\alpha q\right) \geq L(T, q)=-\Upsilon_{M O} q-\alpha \bar{Q}^{2}$ 
because $q \leq \bar{Q}$, and

$$
\begin{aligned}
& \mathcal{T}^{\delta t}(t, q, Z, L) \\
& =L(t, q)+\delta t\left[-\phi_{q} q^{2}\right. \\
& +\sup _{c \in \mathcal{U}}\left[c \left(-\phi_{f}+\left(\gamma^{+}(1)-\gamma^{-}(1)\right) \sigma q+\lambda^{s} p_{s e l l}\left(\Upsilon_{L O}+\Upsilon_{M O}-\kappa L(t, q)\right)\right.\right. \\
& \left.+\lambda^{-} p_{b u y}(Z)\left(V \Upsilon_{L O}-V \Upsilon_{M O}-\kappa L(t, q)\right)\right) \\
& +(1-c)\left(\left(\gamma^{+}(Z)-\gamma^{-}(Z)\right) \sigma q\right. \\
& \left.\left.+\lambda^{+}(Z) p_{\text {sell }}\left(\Upsilon_{L O}+\Upsilon_{M O}-\kappa L(t, q)\right)\right)\right] \\
& \left.+\sum_{K \neq Z}-\kappa L(t, q) G_{Z, K}\right] \\
& \geq L(t, q)-\delta t\left(\max _{i} \gamma^{-}(i) \sigma q+\phi_{q} q^{2}+\phi_{f}\right) \\
& \geq L(t, q)-\delta t\left(\max _{i} \gamma^{-}(i) \sigma \bar{Q}+\phi_{q} \bar{Q}^{2}+\phi_{f}\right)=L(t-\delta t, q) .
\end{aligned}
$$

Together with

$$
\begin{aligned}
\mathcal{M}^{\delta t}(t, q, Z, L) & =\frac{L(t, q)}{1+\kappa} \\
& =\frac{L(t-\delta t, q)+\delta t\left(\max _{i} \gamma^{-}(i) \sigma \bar{Q}+\phi_{q} \bar{Q}^{2}+\phi_{f}\right)}{1+\kappa} \\
& \geq \frac{L(t-\delta t, q)+\kappa L(t-\delta t, q)}{1+\kappa} \\
& =L(t-\delta t, q),
\end{aligned}
$$

we have

$$
\mathcal{S}^{\delta t}(t, q, Z, L) \geq L(t-\delta t, q) .
$$

As $h^{\delta t}(t-\delta t, q, Z)=\mathcal{S}^{\delta t}\left(t, q, Z, h^{\delta t}(t, q, Z)\right)$, we prove by induction that $h^{\delta t}(t, q, Z) \geq L(t, q)$.

\section{A.5. Proof of Theorem 2}

Proof. The proof follows from Corollary 1, Proposition 1, 2, 3, and Barles and Souganidis (1991). 
We define

$$
h_{*}(t, q, Z)=\liminf _{\substack{\delta t \rightarrow 0 \\ t^{\prime} \rightarrow t}} h^{\delta t}(t, q, Z) \quad \text { and } \quad h^{*}(t, q, Z)=\limsup _{\substack{\delta t \rightarrow 0 \\ t^{\prime} \rightarrow t}} h^{\delta t}(t, q, Z)
$$

which are, respectively, lower and upper semi-continuous functions on $[0, T] \times([0, \bar{Q}] \cap \mathbb{Z}) \times\{1,2,3\}$, and inherit the boundedness of $\left\{h^{\delta t}(t, q, Z)\right\}$ by stability from Proposition 2. By definition, we have $h_{*} \leq h^{*}$. We claim that $h_{*}$ and $h^{*}$ are, respectively, a viscosity supersolution and a viscosity subsolution of (31) and (32), then by Corollary 1 (Comparison Principle) we have $h^{*} \leq h_{*}$ and hence the equality. By symmetry, it suffices to show the viscosity supersolution property of $h_{*}$.

Let $(\bar{t}, \bar{q}, \bar{Z}) \in[0, T) \times([0, \bar{Q}] \cap \mathbb{Z}) \times\{1,2,3\}$ and $\varphi \in C_{b}^{1}([0, T] \times([0, \bar{Q}] \cap \mathbb{Z}) \times\{1,2,3\})$ such that $(\bar{t}, \bar{q}, \bar{Z})$ attains the strict global minimum of $h_{*}-\varphi$. Then there exits a sequence $\left\{\left(t_{k}^{\prime}, q_{k}^{\prime}, Z_{k}^{\prime}\right)\right\}_{k} \in$ $[0, T) \times([0, \bar{Q}] \cap \mathbb{Z}) \times\{1,2,3\}$ and $\left\{\delta t_{k}\right\}_{k}$ such that

$$
\begin{aligned}
\left(t_{k}^{\prime}, q_{k}^{\prime}, Z_{k}^{\prime}\right) & \rightarrow(\bar{t}, \bar{q}, \bar{Z}), \\
\delta t_{k} & \rightarrow 0, \\
h^{\delta t} & \rightarrow h_{*}(\bar{t}, \bar{q}, \bar{Z}),
\end{aligned}
$$

and $\left(t_{k}^{\prime}, q_{k}^{\prime}, Z_{k}^{\prime}\right)$ is the global minimizer of $h^{\delta t_{k}}-\varphi$.

Here we restrict $\delta t_{k}$ to satisfy the condition in Proposition 1, so that we apply the monotonicity of $\mathcal{S}^{\delta t}$.

We define $\varepsilon_{k}=\left(h^{\delta t_{k}}-\varphi\right)\left(t_{k}^{\prime}, q_{k}^{\prime}, Z_{k}^{\prime}\right)$. Then by the numerical scheme in 22 and the monotonicity from Proposition 1, we have

$$
\begin{aligned}
\varepsilon_{k}+\varphi\left(t_{k}^{\prime}, q_{k}^{\prime}, Z_{k}^{\prime}\right) & =h^{\delta t_{k}}\left(t_{k}^{\prime}, q_{k}^{\prime}, Z_{k}^{\prime}\right) \\
& =\mathcal{S}^{\delta t_{k}}\left(t_{k}^{\prime}+\delta t_{k}, q_{k}^{\prime}, Z_{k}^{\prime}, h^{\delta t_{k}}\right) \\
& \geq \mathcal{S}^{\delta t_{k}}\left(t_{k}^{\prime}+\delta t_{k}, q_{k}^{\prime}, Z_{k}^{\prime}, \varphi+\varepsilon_{k}\right) \\
& =\mathcal{S}^{\delta t_{k}}\left(t_{k}^{\prime}+\delta t_{k}, q_{k}^{\prime}, Z_{k}^{\prime}, \varphi\right)+\varepsilon_{k} \\
& =\max \left\{\mathcal{T}^{\delta t_{k}}\left(t_{k}^{\prime}+\delta t_{k}, q_{k}^{\prime}, Z_{k}^{\prime}, \varphi\right), \mathcal{M}^{\delta t_{k}}\left(t_{k}^{\prime}+\delta t_{k}, q_{k}^{\prime}, Z_{k}^{\prime}, \varphi\right)\right\}+\varepsilon_{k} .
\end{aligned}
$$

After rearranging, we have

$$
\begin{aligned}
\max \left\{\frac{1}{\delta t_{k}}\left[\mathcal{T}^{\delta t_{k}}\left(t_{k}^{\prime}+\delta t_{k}, q_{k}^{\prime}, Z_{k}^{\prime}, \varphi\right)-\varphi\left(t_{k}^{\prime}, q_{k}^{\prime}, Z_{k}^{\prime}\right)\right]\right. \\
\left.(1+\kappa)\left[\mathcal{M}^{\delta t_{k}}\left(t_{k}^{\prime}+\delta t_{k}, q_{k}^{\prime}, Z_{k}^{\prime}, \varphi\right)-\varphi\left(t_{k}^{\prime}, q_{k}^{\prime}, Z_{k}^{\prime}\right)\right]\right\} \leq 0 .
\end{aligned}
$$


We apply the consistency in Proposition 3 and let $k \rightarrow \infty$, and obtain

$$
\begin{array}{r}
\max \left\{\partial_{t} \varphi-\phi_{q} q^{2}\right. \\
+\sup _{c \in \mathcal{U}}\left[c \left(-\phi_{f}+\left(\gamma^{+}(1)-\gamma^{-}(1)\right) \sigma q+\lambda^{s} p_{\text {sell }}\left(\Upsilon_{L O}+\varphi(t, q-1, Z)-(1+\kappa) \varphi\right)\right.\right. \\
\left.+\lambda^{-} p_{\text {buy }}(Z)\left(V \Upsilon_{L O}+\varphi(t, q+V, Z)-(1+\kappa) \varphi\right)\right) \\
\left.+(1-c)\left(\left(\gamma^{+}(Z)-\gamma^{-}(Z)\right) \sigma q+\lambda^{+}(Z) p_{\text {sell }}\left(\Upsilon_{L O}+\varphi(t, q-1, Z)-(1+\kappa) \varphi\right)\right)\right] \\
\quad+\sum_{K \neq Z}(\varphi(t, q, K)-(1+\kappa) \varphi(t, q, Z)) G_{Z, K}, \\
\left.\quad \varphi(t, q-1, Z)-\Upsilon_{M O}-(1+\kappa) \varphi\right\} \leq 0,
\end{array}
$$

which is the viscosity supersolution property as desired. Therefore we have $h^{*} \leq h_{*}$, and hence $h^{*}=h_{*}$. 